\title{
Basic Gestures as Spatiotemporal Reference Frames for Repetitive Dance/Music Patterns in Samba and Charleston
}

\author{
Marc Leman \& Luiz Naveda \\ Ghent University, Ghent, Belgium
}

THE GOAL OF THE PRESENT STUDY IS TO GAIN BETTER insight into how dancers establish, through dancing, a spatiotemporal reference frame in synchrony with musical cues. With the aim of achieving this, repetitive dance patterns of samba and Charleston were recorded using a three-dimensional motion capture system. Geometric patterns then were extracted from each joint of the dancer's body. The method uses a body-centered reference frame and decomposes the movement into nonorthogonal periodicities that match periods of the musical meter. Musical cues (such as meter and loudness) as well as action-based cues (such as velocity) can be projected onto the patterns, thus providing spatiotemporal reference frames, or 'basic gestures,' for action-perception couplings. Conceptually speaking, the spatiotemporal reference frames control minimum effort points in action-perception couplings. They reside as memory patterns in the mental and/or motor domains, ready to be dynamically transformed in dance movements. The present study raises a number of hypotheses related to spatial cognition that may serve as guiding principles for future dance/music studies.

Received November 2, 2009, accepted May 6, 2010.

Key words: basic gesture, dance, spatiotemporal reference, samba, Charleston

$\mathrm{P}$ OPULAR DANCE STYLES OFTEN ARE characterized by repetitive dance patterns that typically occur in synchrony with structures of the musical meter. In what follows, examples of repetitive dance patterns are taken from samba and Charleston dances. They can be seen as genuine instantiations of a large collection of dance typologies (often called popular, folk, or traditional dances) in which repetitive movements form an important part of the choreography.

In this paper, we explore the idea that repetitive dance patterns are based on spatiotemporal reference frames — called basic gestures - that capture repetitive dance patterns in terms of a simple geometry onto which cues from music (e.g., meter, loudness) and body (e.g., speed) can be projected. Through the concept of basic gestures, we aim at developing a cross-modal approach that results in a representation that may contribute to a better understanding of the tight coupling of dance and music, and, in more general terms, the coupling of action and perception.

Dancing to music can be related to a general framework of embodied music cognition (Leman, 2007). In repetitive dances, this includes body movements that occur in synchronization (and entrainment), as well as in counterpoint with, metrical properties of the music, such as the tempo and pulse of the beat, and multiples and divisions of the beat. In short, the embodied viewpoint entails the idea that dancing to music is an activity that strongly relies on the coupling of action and perception, and that the human body plays an important role as a mediator that couples subjective experiences with the physical environment.

When studying dance patterns in relation to music, there is a need for detailed descriptions. Analysis techniques have been developed that capture features of dance patterns as objective time-varying structural descriptors, such as the quantity of movement and the contraction index in video analysis (Camurri, 2002; Camurri, Lagerlof, \& Volpe, 2003; Camurri, Mazzarino, \& Volpe, 2004; Glowinski, Camurri, Chiorri, Mazzarino, \& Volpe, 2009; Guedes, 2006). However, these time-varying structural descriptors say little about the dancer's interaction with music and space. To uncover these relationships, a correlation analysis of the time-varying structural descriptors of movement in relation to musical features is needed, but this is difficult if no cross-modal representations of the coupling between these domains are available.

\footnotetext{
${ }^{1}$ The contraction index is inspired by Laban's notion of a kinesphere (Laban \& Lawrence, 1947; Laban \& Ullmann, 1966, 1980), which is a hypothetical space defined by the extremities of the human body posture, reminiscent to Leonardo da Vinci's Vitruvian man (Counsell, 2006). The contraction index is calculated as the ratio between the area of the object's silhouette and the area of object's bounding rectangle. As the dancer moves, the parameters of the contraction index move along as time-varying variables.
} 
In parallel with the development of video analysis platforms and tools, some empirical studies on dance have focused on the extraction of time-varying variables from motion capture systems and motion sensing systems (Bevilacqua, Ridenour, \& Cuccia, 2002; De Bruyn, Leman, Moelants, \& Demey, 2009; Eerola, Luck, \& Toiviainen, 2006; Shiratori, Nakazawa, \& Ikeuchi, 2003; Yamamoto \& Fujinami, 2008). So far, mainly aspects of movement synchronization and movement intensity have been considered, and little attention has been devoted to the spatiotemporal development of the dance patterns. Many researchers therefore will agree that the study of dance/ music couplings is still in an initial stage, and that there is a lack of concepts and tools that can define the coupling of dance and music within a unified framework.

We believe that the concept of basic gesture may offer a particular contribution to a spatiotemporal framework that allows the study of repetitive dance patterns in close relation to musical cues. In this context, it is important to mention that the concept of basic gesture is intended to provide a viewpoint on action-perception couplings that is conceptual rather than physiological. As such, it may be related to three different representational domains. First, a basic gesture may be related to the mental domain, where it would function as a mental representation, possibly defined as an image, of a spatiotemporal reference frame for repetitive dance patterns. Second, a basic gesture may be related to the motor domain, where it would function as a motor schema; that is, a frame of reference or disposition for motor activity in response to auditory input. The difference between mental representation and motor representation is that the latter would typically require less memory load at the moment when the intentional action is physically carried out. And finally, a basic gesture may be related to the executed dance pattern itself, or even better, to a trajectory of the body movement as physically deployed in space and time. These three viewpoints play a role in our current thinking about musical gestures (see Godøy \& Leman, 2010). The idea that a gesture may manifest itself 'out of time'-that is, as a representation of a reference frame for action-perception, is crucial to explain why we speak about 'gesture' and not just about 'body movement' (Leman \& Godøy, 2010).

Given the framework described above, it is possible to adopt an empirical approach in which basic gestures are reconstructed from physically deployed repetitive dance patterns. In this approach, a physical repetitive dance pattern is seen as the execution of a mental and/or motor pattern, whose particularity lies in the fact that it is the representation of a frame of reference that aligns body movement with musical cues.

Thus, in the present study, we focus on how dancers deploy repetitive dance patterns in synchrony and counterpoint with musical cues. A method is described that allows the re-construction of basic gestures from these repetitive dance patterns. The method is based on a number of assumptions (outlined below) that may serve as guiding principles for future dance/music studies. Although our main target is to show that a basic gesture can be represented as a geometrical shape onto which music cues (e.g., related to timing, loudness) can be projected, it will be straightforward to extend the method to action cues (e.g., speed) as well.

By concentrating on the analysis of individual dancers, we hope to be able to develop an approach that represents both the general and the particular: the generality is related to the description of a global concept of basic gesture as representational structure of a spatiotemporal reference frame for action-perception, whereas the particularity is related to the assumption that musical styles have their proper reference frames, and that dancers have their proper gestural characteristics.

\section{The Concept of Basic Gesture}

The concept of a basic gesture can be traced back to Becking (1928), who described the general form of sympathetic movements in response to music as a kind of geometric shape that summarizes the trajectories of a repetitive pattern (Becking, 1928; Nettheim \& Becking, 1996).

Becking's concept of basic gesture is still relevant to studies in which a motion capture system measures kinematic aspects of dance movements. Starting from physical movements, a basic gesture can then technically be defined as the three-dimensional movement pattern of a body part during one period of a repetitive dance sequence. One should add that the measurement by a motion capture system is actually limited to the measurement of fixed points (so-called markers) on the body but doesn't measure the entire body movement. Furthermore, two considerations concerning shape and timing should be taken into account, which somehow frame (and thus confine) our current concept of gesture. The first consideration is that the shape of a basic gesture (more specifically, the three-dimensional trajectory of one period of a repetitive movement of a fixed point on the body) will be such that its starting point and ending point will be connected. Otherwise, it cannot be considered to be repetitive. The second consideration takes into account the timing of the gesture, which, we assume, is related to the natural frequencies (or eigenfrequencies) at which body parts tend to move. For example, repetitive movements of legs during walking can characterize a basic movement, because these movements are repetitive and their timing is related to the biomechanics of the human body (Styns, van Noorden, Moelants, \& Leman, 2007). The 
basic gesture is called 'basic' because it appears as an ideal reference frame for matching spatial and musical cues in a movement configuration that requires little effort to maintain its repetitive character (Van Noorden, 2010). The situation is similar to a pendulum, where the swaying can be maintained with little input force, provided that the period of the latter fits with the resonance frequency (the eigenfrequency) of the pendulum. The reference frame would specify that the extremities of the pendulum movement would correspond to the beat of the music. There are several studies on tapping along with music (Van Noorden \& Moelants, 1999), walking on music (Styns et al., 2007), spontaneous movement without music (MacDougall \& Moore, 2005), and music-driven group synchronization (De Bruyn, Leman, \& Moelants, 2009; Van Noorden \& De Bruyn, 2009) that suggest that this resonance frequency lies in the vicinity of $2 \mathrm{~Hz}$ (which equals 120 beats per minute (bpm). However, there is a broad range of musically relevant beat frequencies from 30 to $240 \mathrm{bpm}$ (London, 2006) that may be considered relevant. In short, basic gestures are assumed to have a simple geometry (a three-dimensional line that is connected), and a simple timing basis (related to the body's resonance frequency). Their geometric description is called a Becking curve.

\section{Basic Gestures and Musical Meter}

Styns et al. (2007) explored the concept of basic gesture in studies on how people walk to the beat of the music. He focused on the speed of walking in relation to the musical tempo (i.e., beats per second). In a series of studies on samba dance and music (Naveda \& Leman, 2008a, 2008b, 2009), this concept of basic gesture was further developed and was related to the hierarchical structure of musical meter. The latter consists of multiples and divisions of the beat period. The approach was based on the idea that movement can be studied by using the musical meter as a gauge. It introduced a crossmodal method in which a metrical grid (defined with respect to multiplications and divisions of the musical beat) was used to select body movement periodicities (see Figure 1). The method uses a heuristic based on periodicity transforms (Sethares \& Staley, 1999) that produces a map of repetitive patterns in dance similar to the Becking curves (Becking, 1928). This leads to a spatial representation of basic gestures, onto which additional indication of the timing of the metrical grid can be projected. It was shown that basic gestures can be extracted straightforwardly from repetitive dance patterns, and optimal solutions are obtained when the musical metrical grid fits with the repetitive period of the dance patterns. As such, the basic gestures can be related to the time points of the meter (double beat, beat, half beat, quarter beat, etc.), thus providing a spatiotemporal representation of repetitive dance gestures.

Using this analysis approach, Naveda and Leman (2009) found evidence that (binary) periodicities in the gestures of the samba dancer may disambiguate the metrical ambiguity (binary versus ternary groupings of the beat) in samba music. The process of corporealbased disambiguation can thereby be seen as a meaning formation process that is rooted in the deployment of the body, rather than by mere mental processing of sonic structures (Leman, 2007; Phillips-Silver \& Trainor, 2007).

However, the approach described in Naveda and Leman (2009) had two major limitations. First, it was based on the analysis of video recordings of dance, and therefore, the extracted patterns were two-dimensional. Second, the method considered body movement (and basic gestures) from the viewpoint of an absolute reference framework that is defined by the video recording position. The latter implies that from a different observation position, different basic gestures are extracted. Instead, what is needed is an analysis that considers (i) three-dimensional representations of basic gestures, and (ii) representations that are invariant with respect to the observation position. The shift from two dimensions to three dimensions can be obtained with a motion capture system. The shift from absolute reference framework to a relative reference framework, however, affects the representational nature of the basic gesture in the sense that it changes the viewpoint from observer to dancer. The relative framework is therefore called a body-centered reference framework, and it refers to the peripersonal space (i.e., the space surrounding our body that can be reached by our limbs) around the dancer.

The following three assumptions summarize the starting position for our current study of basic gestures. The first assumption states that movements of all body markers in free space (viewpoint of the observer) can be related to the geometrical centroid of the dancing body, so that all movement trajectories can be linked to the dancer's body-centered frame of reference. The second assumption states that dance movements are consequences or inductors of the metrical structure of the music, and it implies that a musical metrical grid can be used as a gauge to look

${ }^{2}$ Joints (and short names) selected for the calculation of basic gestures: root (root), left hip (lhip), left knee (lknee), left toe (ltoe), right hip (rhip), right knee (rknee), right toe (rtoe), mid-torso (midtorso), neck (neck), head (head), left shoulder (lshoulder), left elbow (lelbow), left finger (lfinger), right shoulder (rshoulder), right elbow (relbow), right finger (rfinger). 


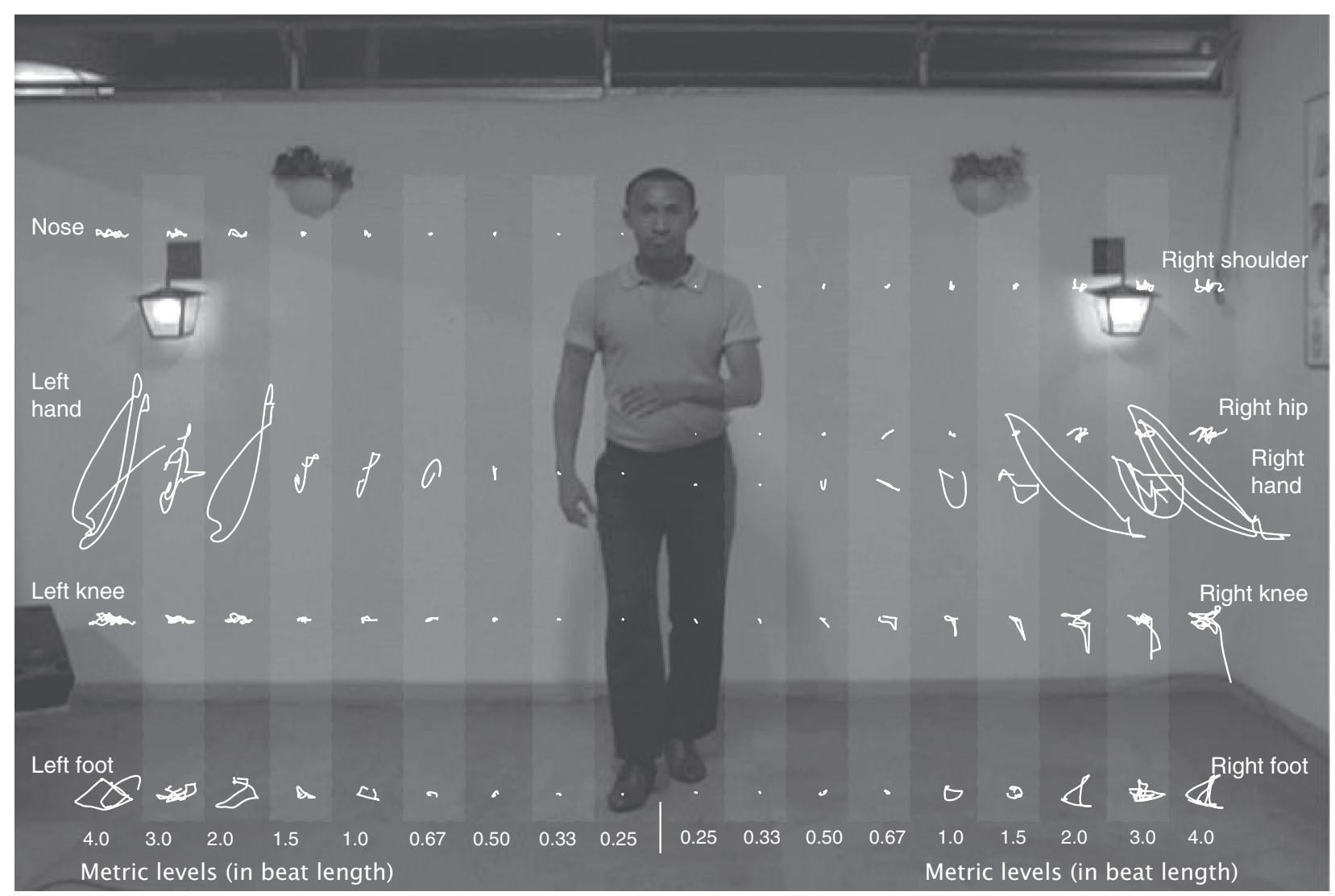

FIGURE 1. Samba dancer and Becking curves associated to different body parts (Naveda \& Leman, 2009, p. 279). The Becking curves represent the shapes of basic gestures, of which the period corresponds to the period of the metric grid (horizontal). Note that the Becking curves are not always closed, which is due to the method used in Naveda and Leman (2009).

at body movement. The third assumption states that repetitive dance patterns rely on reference frames that are related to the eigenfrequency of the body, and for that reason, these reference frames require less effort. What follows is a description of a method that takes these assumptions into account.

\section{Method}

\section{Experimental Setup}

The dancers wore a dance suit with 34 reflective markers attached to it; the markers provided the point-set representation of body morphology. The markers were placed on: head (3), upper arms $(3+3)$, upper back (3), hips $(4)$, hands $(3+3)$, thighs/knee $(2+2)$, shins $(1+1)$, and feet/toe $(3+3)$. The stimulus used to perform the samba dances (professional dancer and students) was composed of looped samples of a samba percussion ensemble (surdo, tamborim, and caxixi). The samples were recorded from professional samba musicians in Brazil, using a multitrack recorder. The stimulus used to accompany Charleston dances was composed of phrases of Charleston music ("Novelty Charleston," Titanic Ensemble). The bpm was 90 for the samba stimulus and 111 for the Charleston stimulus.

The movement recordings were recorded using an Optitrack system (Natural Point) composed of 12 cameras positioned around a squared aluminum structure $(6 \times 6$ square meters). The duration of each take was approximately $60 \mathrm{~s}$ at a frame rate of $100 \mathrm{~Hz}$. The recordings were synchronized with audio using movement cues performed by the subject in synchrony in predefined audio onsets played at the beginning of the audio stimulus.

SUBJECTS

Two professional dancers/teachers and one dance student participated in the experiments (all females). The Charleston dancer was a professional Dutch dancer/ teacher who specialized in old and traditional dances and had several years of experience in performance and 


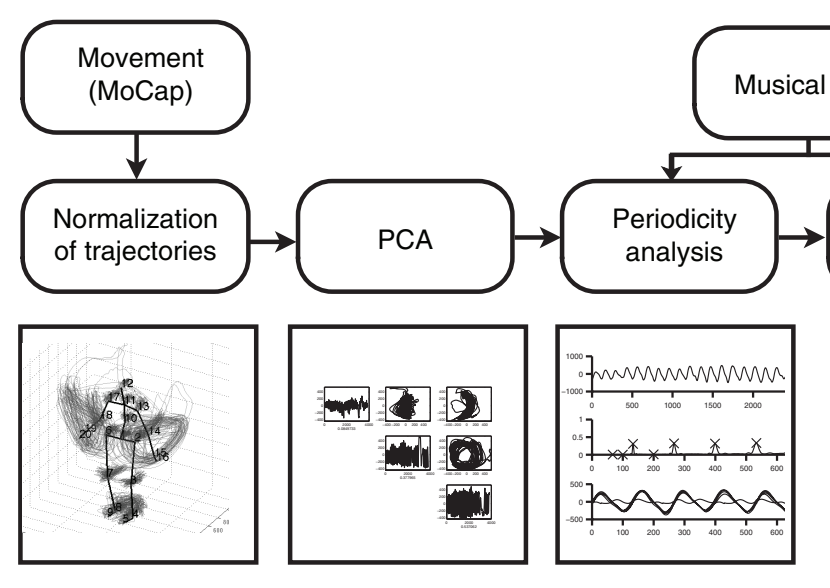

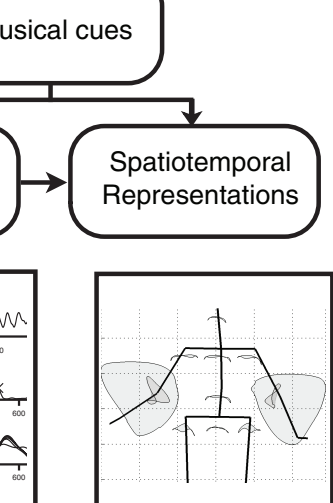

Basic gesture

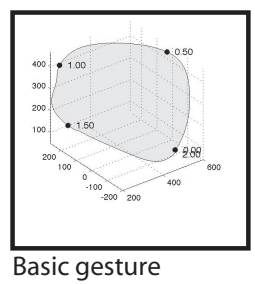

and metrical points

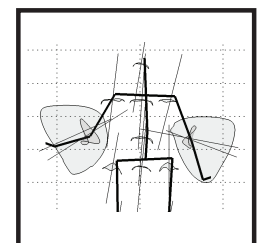

Gesture axis
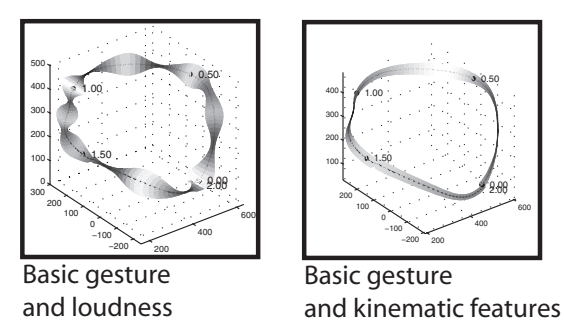

FIGURE 2. Illustrative flow chart describing the methodology used in the data analysis. On top, the movements are recorded by a motion capture system (MoCap) and the musical cues are presented. The second row from top displays the processing flow. The figures in rectangular boxes show the resulting images at each processing stage.

teaching. She performed dances in basic Charleston style. The samba dancer was a Brazilian female dancer/teacher who specialized in Afro-Brazilian dances and had several years of dance experience in performance and teaching. She performed dances in the "Samba-no-pé" style, which is the main substyle of samba dances. The student of samba dance was a Belgian female dance amateur, with 1.5 years of experience in samba dance (lessons). The lessons were taken with the same professional dancer who danced in this experiment.

\section{TASK}

The dance excerpts used in this study had different lengths. The dancers were asked to perform examples of the basic dance forms within a defined circular area (diameter $=4 \mathrm{~m}$ ) in a relatively isolated environment. They were asked to avoid unnecessary turns in the body orientation (frontal performance, in relation to a single direction).

\section{MATERIAL}

The recording sessions were edited and exported as C3D files using the software ARENA (Optitrack/Natural Point). The sequences were imported into Matlab using the MoCap toolbox (Toiviainen \& Burger, 2010). The calculation of body-centered segment positions, filtering of raw vectors, normalization, and part of the visualization functions, were also based on the MoCap Toolbox.

\section{Data Analysis}

The data analysis consisted of a chain of processes that involve normalization, principal component analysis, and periodicity detection (see Figure 2). First, the trajectories in free space were normalized in order to obtain movement data in the peri-personal space. Then, a principal component analysis (PCA) was applied to the data in order to determine the dimension (or coordinate) that captures the largest variance of the gesture's trajectories. In this dimension, a periodicity analysis is carried out, using the musical grid from the musical stimulus as a guide for searching the periodicity. The shape and periodicity of the gesture was then reconstructed from the other dimensions onto which musical cues and action cues could be projected. Figure 2 shows a schematic overview of the processing and its results.

\section{NORMALIZATION OF TRAJECTORIES}

The raw data from the motion caption recordings appear in an arbitrary coordinate system $(\mathrm{X}, \mathrm{Y}, \mathrm{Z})$ with a fixed reference point that was defined during the setup of the 


\section{Normalization of trajectories: professional samba dancer}

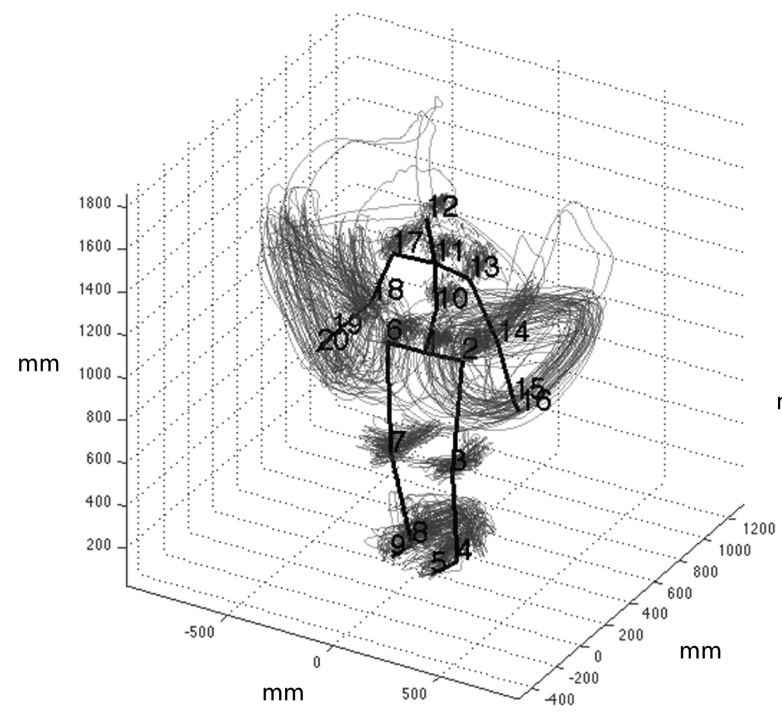

a - raw trajectories

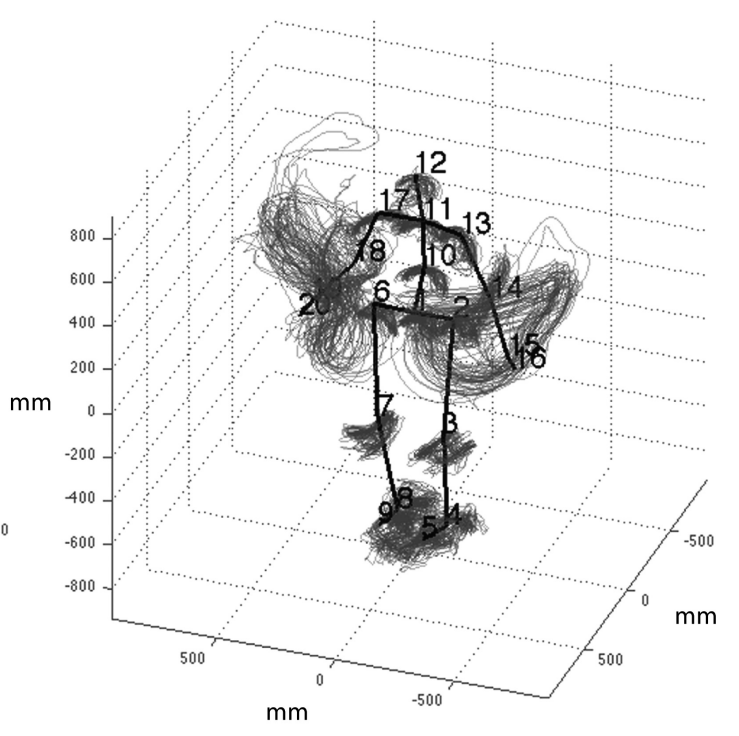

b - normalized trajectories

FIGURE 3. Movement trajectories of the samba dance for 16 joints. (a) The movement trajectories of repetitive dance patterns in free space (raw data). (b) The normalized movement trajectories.

motion capture system. From these raw data, bodycentered representations could be obtained by relating the recorded trajectories of the joints of the body parts to a single point on the dancer's body. The point of reference was defined as the centroid of the markers on the body. In addition, the orientation was defined as frontal with respect to the left and right hips. The trajectories of all joints was then related to that moving body point, rather than to a fixed arbitrary reference point. This operation, called normalization, resets the coordinates of the joint positions, rotations (turning around of the body in physical space), and translation (displacement of the body in space) for each frame of the recording.

Figure 3 a shows the raw trajectories of repetitive movements of samba dancing, which become visibly less dispersed after normalization in Figure 3b. The figure shows movement trajectories in the peripersonal space of the dancer. The general form of these trajectories is ordered. This suggests the existence of redundant patterns of movement that can be perceived as simplified geometries, or Becking curves, such as circle-like figures.

PRINCIPAL COMPONENT ANALYSIS (PCA)

After normalization, we apply a PCA to the trajectory of each joint. This was done in order to find the dimension where the movement was largest. For each joint, the best perspective for further periodicity analysis was then obtained by a rotation of the trajectory, using the eigenvectors of the PCA analysis. Figure 4 shows the original trajectory description of a repetitive gesture of the hand, measured by means of the joint position of the hand (derived from three markers placed on the hand) and the rotated version of the same trajectories, oriented to display the largest movements in the component dimensions. One can observe that the repetitive pattern occurs in a slightly curved plane, whose main axes are defined by the (two main) principal components.

\section{PERIODICITY TRANSFORM}

To extract the period and shape of a basic gesture from each (PCA-rotated) movement trajectory of a joint, we decomposed the trajectory into a set of basic periodic bases. This decomposition is based on the periodicity transform (Sethares \& Staley, 1999), which finds periodicities and bases of periodicities in the original signal, together with respective measures of energy (also called: norm). Unlike other methods such as Fourier or Wavelet transforms, the periodicity transform (PT) does not make any assumptions about the shape of the basic gesture (see discussion in Naveda \& Leman, 2009, p. 260). However, the results of periodic decomposition are nonorthogonal, which implies that a given periodic basis obtained from a periodicity transform projection is not independent from other periodic bases obtained from the same trajectories (such as in Fourier or wavelet transforms). Different heuristics, different orders of projections, 
PCA analysis: professional samba dancer (right hand)

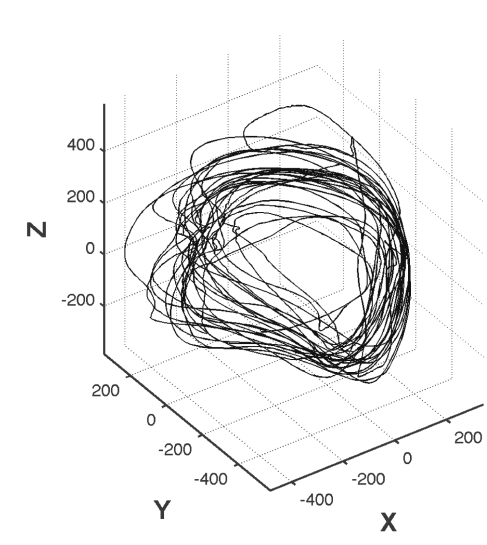

a - Original trajectory

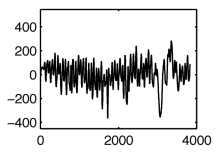

Variance: 0.08

3rd component

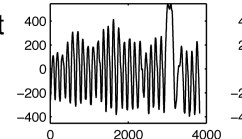

Variance: 0.37

2nd component

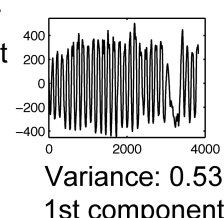

b - PCA analysis: components and variance

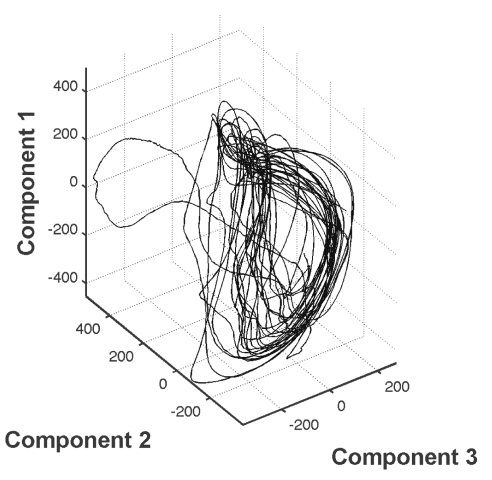

c - Rotated trajectory

FIGURE 4. Movement trajectories of the right hand. (a) The original movement trajectory in a coordinate system that is system dependent. (b) The PCA components and variances (ratio of the total variance). (c) The rotated movement trajectory, which is dependent on the variability of the movement (axes display units in $\mathrm{mm}$ ).

and subtractions of basic period patterns from the signal will lead to different results.

A proper heuristic for the decomposition of repetitive dance patterns can be based on the assumption that musical meter can be used as a gauge to look at the periodicity in dance patterns. In practice, each metrical unit (i.e., the beat, and its multiples and divisions) has a duration, or time period, which defines a sieve (a small time window in the vicinity of that time period), through which we look at the repetitive dance pattern in order to apply the periodicity transform. The period (and corresponding shape) of the repetitive dance pattern is extracted using the Any-Route heuristic (Naveda \& Leman, 2009), which uses a strategy in which each sievebased search starts from the original signal. Alternatively, one could use a heuristic where the most prominent period is first extracted from the signal, and less prominent periods are subsequently extracted from the residues of that signal (as in the Best-Route heuristic in Naveda \& Leman, 2009). Owing to the fact that the Any-Route heuristic starts from the original signal for each period of the metrical grid, this heuristic will find the maximal shape of a particular meter-related period in the dance pattern. The Any-Route heuristic provides a description of the metrical ambiguity of the original signal.

The periodicity analysis, using the periodicity transform, is then applied to each dimension that constitutes the movement trajectories. We thereby assume that the dimension with more variability in the movement trajectory (i.e., larger movements) should guide the periodicity analysis towards the other two dimensions. By using this approach, the detection of the periodicities in the other two dimensions is somehow forced.

It is important to consider that this approach entails two assumptions that influence the detection of periodicities in the PCA-transformed trajectories. The first assumption is that it looks only for periods in the repetitive dance pattern that belongs to the metrical structure of the music. The second assumption is that it extracts the period from the dimension where the repetitive dance pattern displays the largest movements. The periodicities in this dimension are then used to retrieve periodic patterns in other dimensions, so that a basic three-dimensional gesture (consisting of period and shape) can be reconstructed.

Figure 5 displays one example of the periodicity analysis (Any-Route heuristics), applied to the first principal component of the hand trajectory (from the same trajectory described in Figure 4).

The top graph in Figure 5 displays the original signal in the dimension where the variability of the movement trajectory is largest. The second graph displays the metric grid (dashed lines) and the profile and peaks of energy of periodicities (periodicity/signal energy ratio), which are computed using the periodicity transform over all periods. The lower graphs show all the bases of the periodicities selected. These bases reflect the metrical ambiguity of the signal (as an outcome of the non-orthogonal method). Note also that in this example, the four-beat basis does not add any new information to the two-beat basis. 
Periodicity analysis :

Samba - right hand - 1st component/ heuristic Any-route

a) Signal

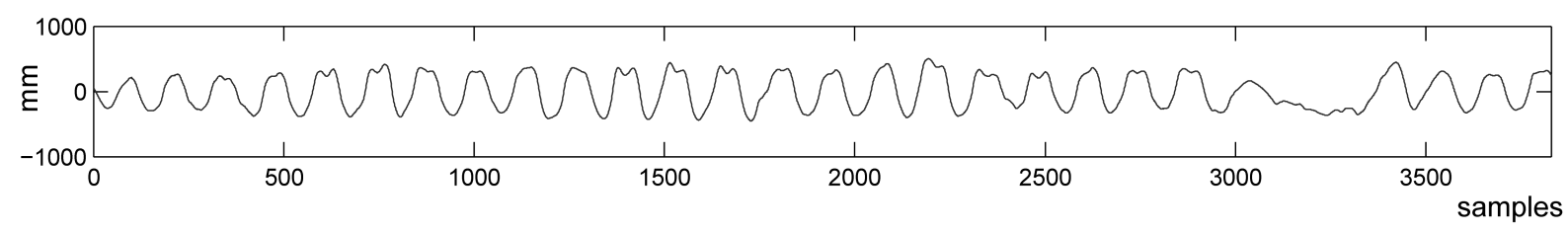

b) Energy and Metric levels

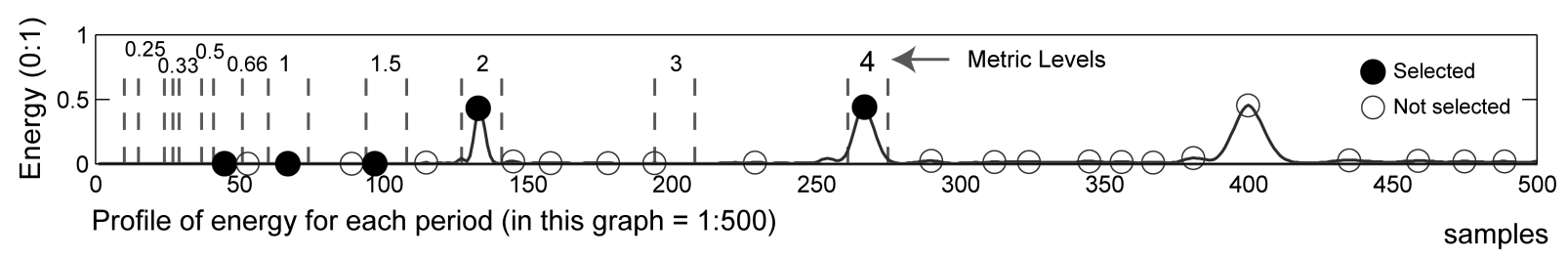

c) Bases
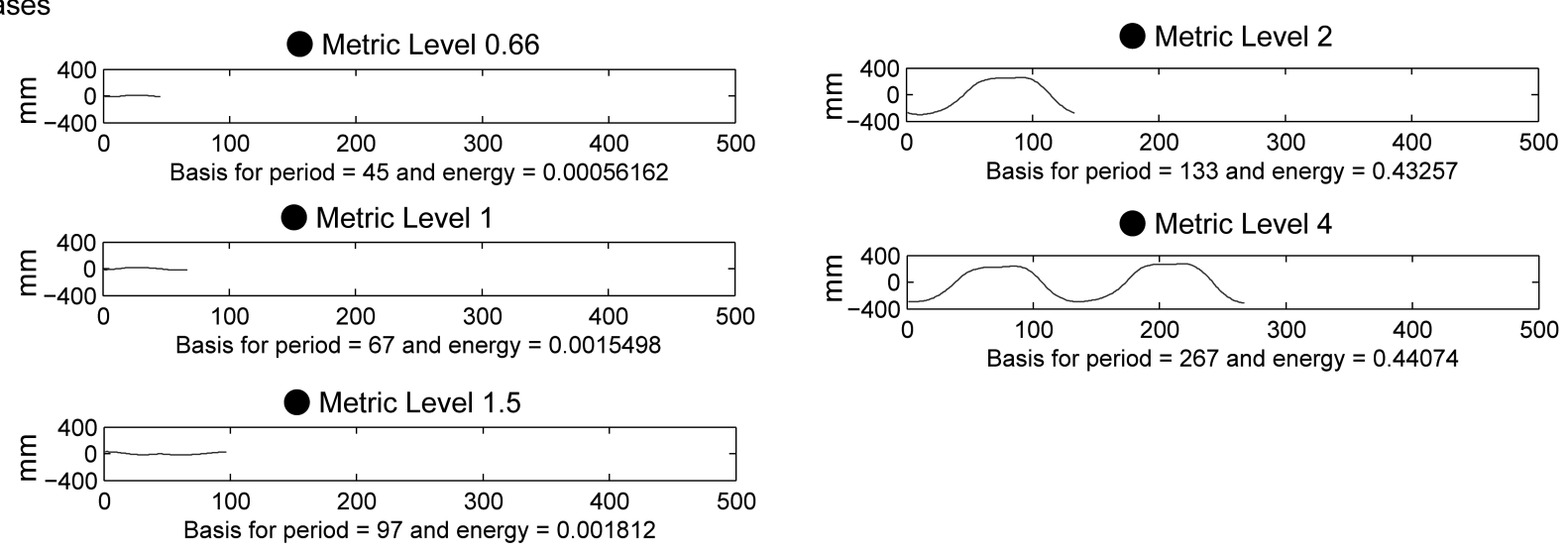

FIGURE 5. Periodicity analysis applied to the dimension where the variability of the movement trajectory is largest. (a) Top panel: the original signal (here: a displacement of a body part represented in one single dimension). (b) Below top panel: periodicity analysis with indication of the metrical grid. (c) Bottom panels: extracted bases, corresponding to the selected periods of $0.66,1,1.5,2$, and 4 times the beat. According to the Any-Route heuristic, each shape is extracted from the original signal.

Figure $6 \mathrm{a}$ and Figure $6 \mathrm{~b}$ show the result of a periodicity analysis for a samba dance and a Charleston dance pattern. The dancer is represented as a stick figure. For each body part, the percentages of the variances are graphically shown, related to (i) the peripersonal space where the $\mathrm{X}$ (left-right), Y (up-down), and Z (back-front) dimensions are shown, and (ii) the principal components of each joint, which reveal the dimensionality of the joints where the gesture is largest. The last column (iii) shows the profile of energies of each extracted metrical period (number 1 stands for the beat), which is defined as the ratio between periodicity and signal energies. This profile indicates which level of the musical meter is likely to be the fundamental period of the movement. The algorithm is defined in such a way that the period with the highest energy value is selected as the period of the basic gesture, of which geometries are shown on the stick figure (see next sections for further explanation of how these geometries are obtained). Note the differences between samba and Charleston: in samba, the basic gesture is two-beat (a four-beat basis has the same value), whereas in Charleston, the basic gesture is four-beat (highest value). The figure also provides information about the direction of the movements for each joint; for example, the tendency for diagonal movements in the hands of the samba dancer (rfinger) or the tendency for left-right movements in the hands of the Charleston dancer. The data suggest that in Charleston dance, basic gestures evoke a single dimension (X: left-right, Y: up-down, Z: back-front), whereas in samba dance, basic gestures evoke two dimensions. 
Variances and periodicities : samba and Charleston

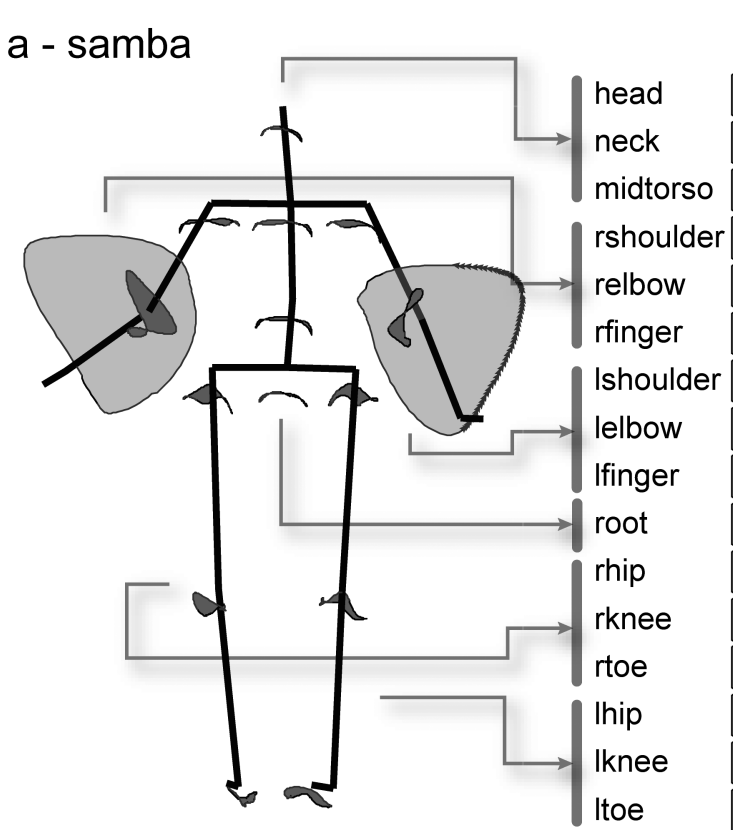

b - Charleston

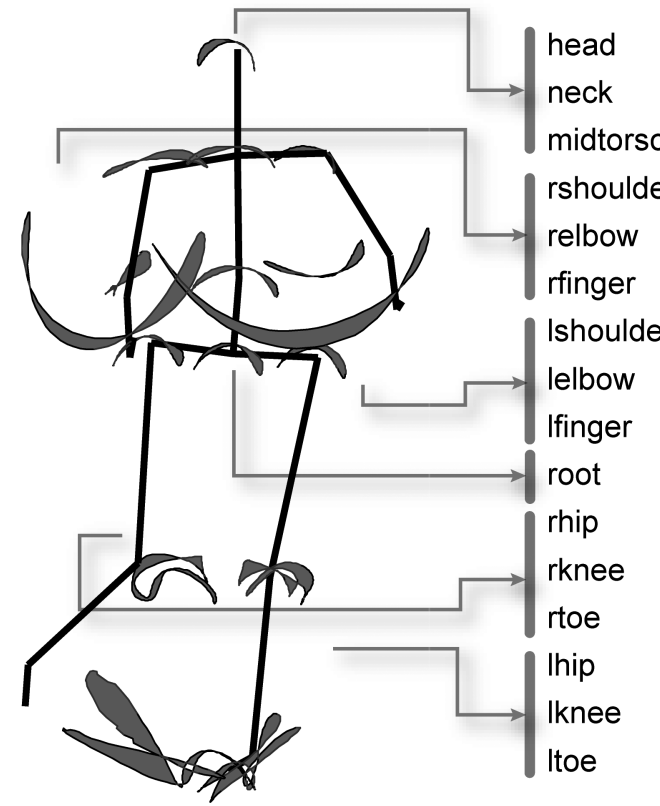

Dimensions

(variance 0:1)

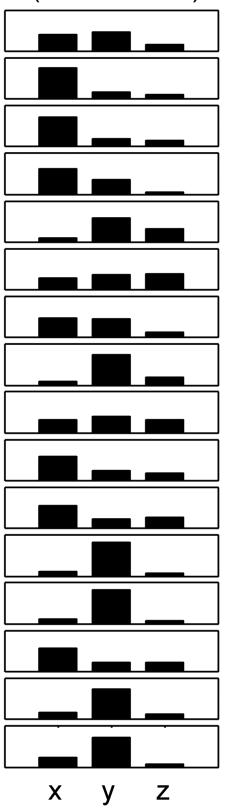

Dimensions (variance 0:1)
PCA components (ratio $=0: 1)$

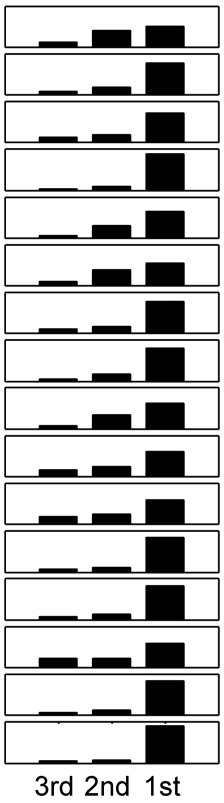

PCA components (ratio $=0: 1$ )

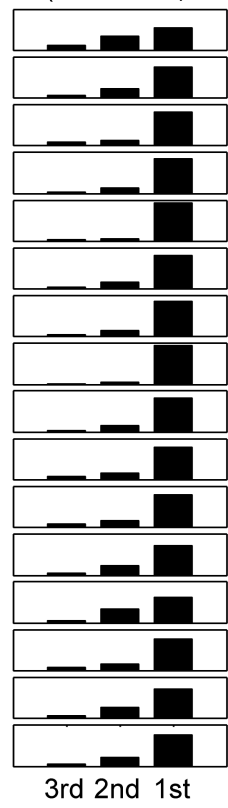

Periodicity Trans.

energy ratio $=0: 1$

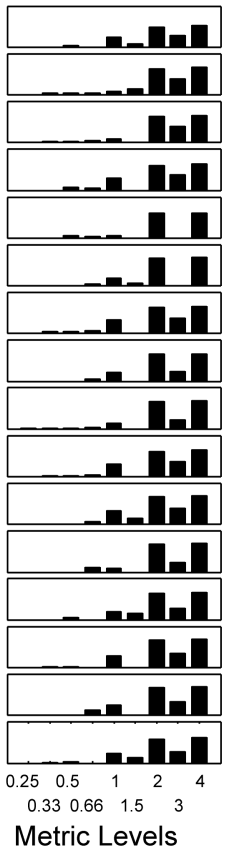

Periodicity Trans (energy ratio $=0: 1$ )

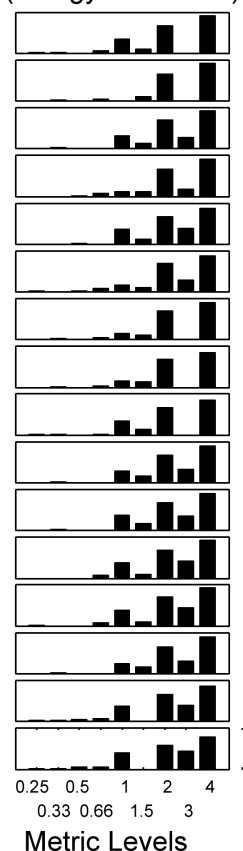

FIGURE 6(a and b). Stick figure (and basic gestures) with (i) levels of original variance, (ii) levels of variance after PCA, and (iii) energy ratio for periodicities, for both samba (a), and Charleston (b). The level of variance is defined as the proportion of the variance of each dimension with respect to the sum of variances of all three dimensions. The energy ratio indicates an average of the energy in each dimension, calculated as the norm or inner product of the periodic pattern with the signal. With the Any-Route algorithm, periodicities at one level may include periodicities found at other levels. 


\section{Basic gesture and metrical grid : professional samba dancer}
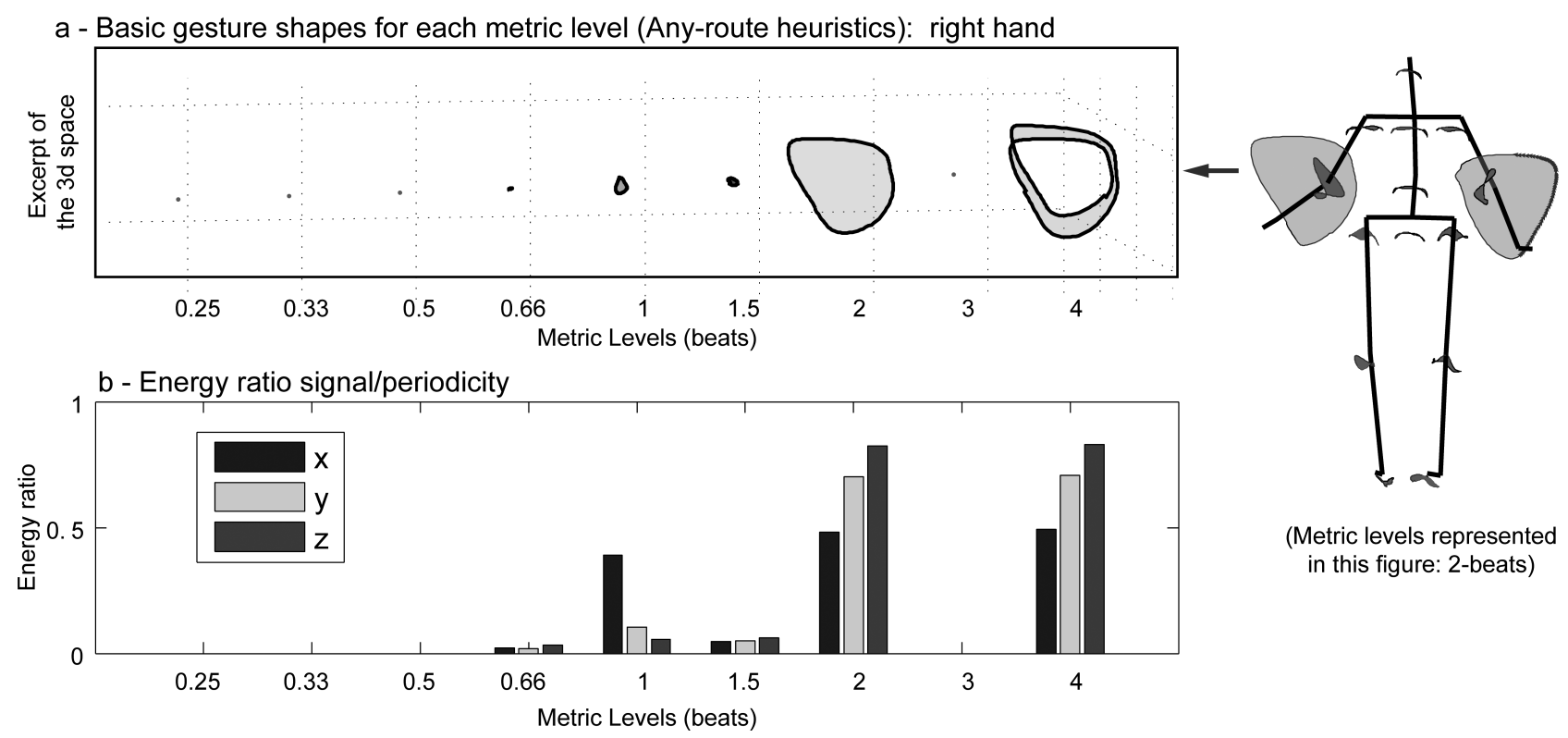

(Metric levels represented in this figure: 2-beats)

FIGURE $7(\mathbf{a}$ and $\mathbf{b})$. The top panel shows the basic gestures of the hand movement shown on a metrical grid. The numbers below each shape define the metrical grid in terms of the musical beat, of which the period is defined as the unit. The bottom panel displays the evidence for the periodicity decomposition showing the energy ratio for each dimension of each basic gesture after the PCA and periodicity analysis.

\section{Analysis and Discussion}

RECONSTRUCTION OF A BASIC GESTURE

Based on the analysis method described above, it is now possible to reconstruct the basic gestures. Figure $7 \mathrm{a}$ shows the basic gestures that can be extracted from the repetitive movement pattern of Figure 5. The gestures are shown on a metrical grid, representing metric levels of 0.25 to 4 times the duration of the beat. The amplitude of the gesture shapes shows the level of (the nonorthogonal periodicities') response, for each metrical grid. This can be interpreted as the likelihood of the basic gesture occurring at that metrical unit. The size of each shape thus indicates how likely it is that this shape underlies the movement trajectory. As shown in Figure 7 (top), the most likely period corresponds to two beats. This gives the most compact gesture, since the gestures at metric level 4 already contain repetitions of this basic gesture and almost the same energy ratio (bottom).

\section{Basic Gestures of Samba and Charleston}

The above method enables us to compare the repetitive dance patterns of samba and Charleston dance. Figure 8 (samba) and Figure 9 (Charleston) show 16 basic gestures selected from 20 original joint trajectories in connection to a stick figure that is taken from a random frame of the dance sequence. These joints are extracted from a triangulation of the skeleton of 34 markers, described in the methods section (see Toiviainen \& Burger, 2010, for more information about this process).

In samba, the gestures are represented at a period of two times the duration of the beat (two-beats metric layer), which seems to be the fundamental element of the metrical gesture palette (see Figure 7) and also the characteristic bar level of samba music. The basic gestures of the hands are more pronounced than the gestures of the other body parts; this does not necessarily mean they are more perceptible or important for the dancer. Hands move more but represent a small part of the total mass of the dancer's body. Less pronounced movements, such as the head and torso, are linked with a considerable proportion of the body mass. Movement of the torso and head in the samba dance exhibits a considerable similarity concerning shape and amplitude, which may be explained by the morphological constrains of these parts.

The basic gesture of the Charleston dance is displayed in Figure 9. Even with the normalization of trajectories in relation to the body, we observe that Charleston gestures are more extended than samba gestures. The areas inside the basic gestures indicate how the extension differs in terms of the occupation of space. The space inside of the Charleston hand gestures is thinner than the space inside the samba hand gestures, and paths are almost 
Basic gesture: professional samba dancer
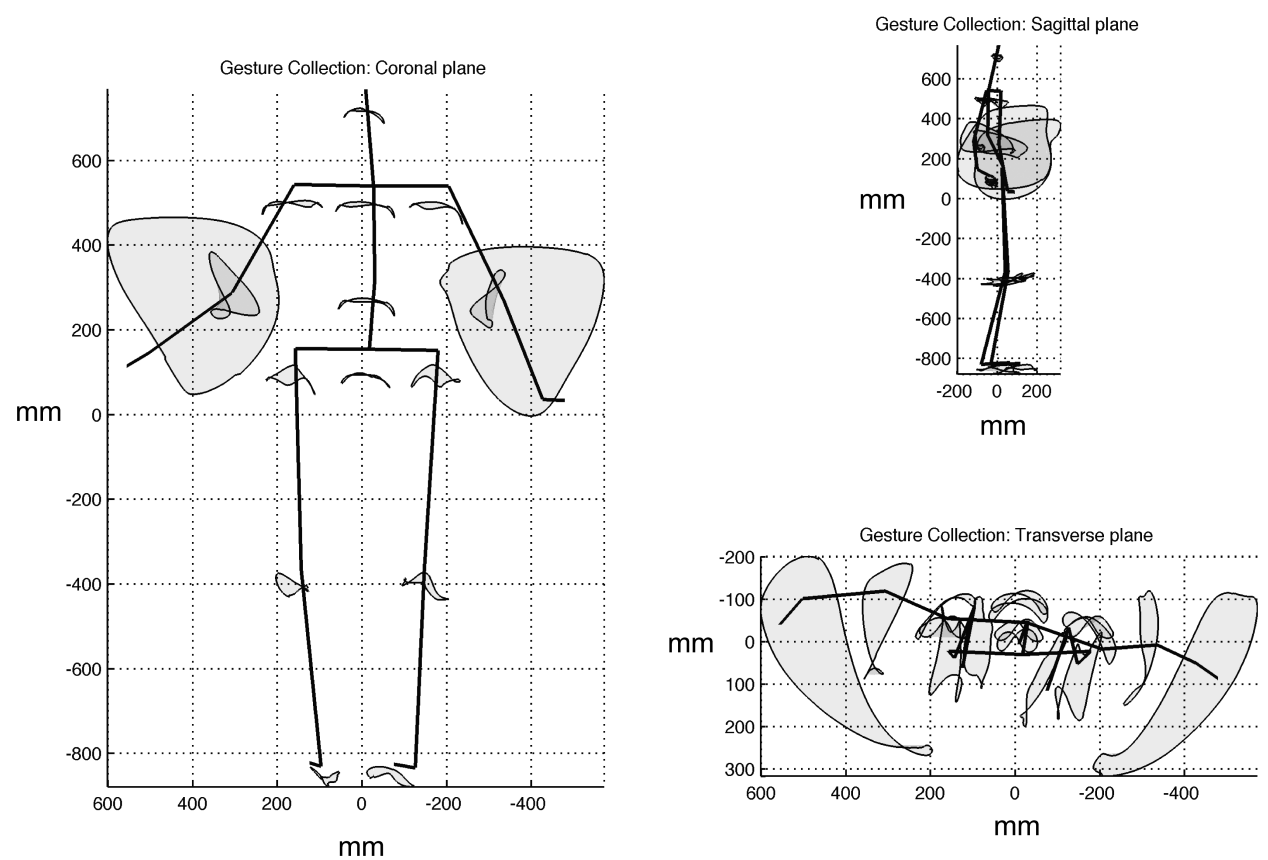

FIGURE 8. Basic samba gestures for different body joints. The metric level is two-beat. The internal area of each shape is indicated by a transparent gray value so that superposition of shapes in an angle of vision can be intuitively perceived (a gesture shape behind another will appear darker than a gesture shape in the front). The representation of the dancers' body (stick figure) is taken from a random frame (it only represents a snapshot of the dancer's body in time).

Basic gesture: professional Charleston dancer

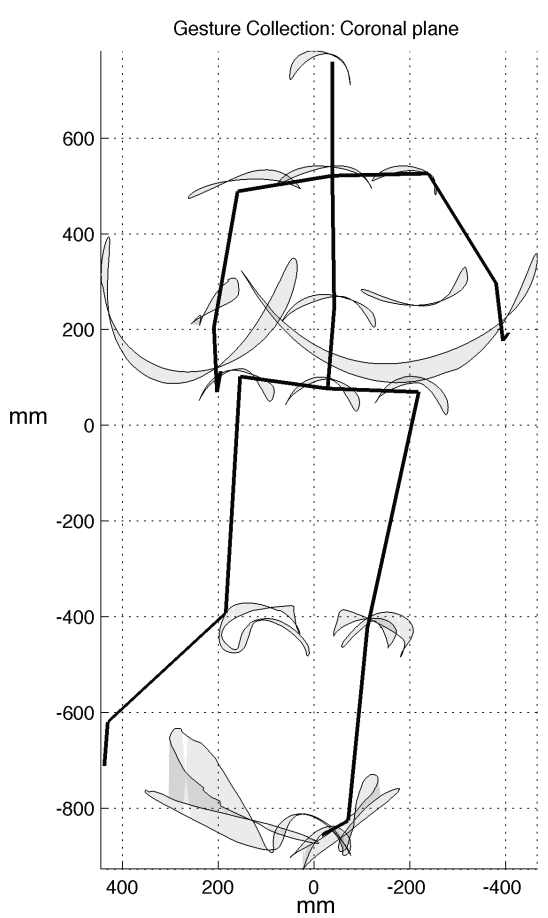

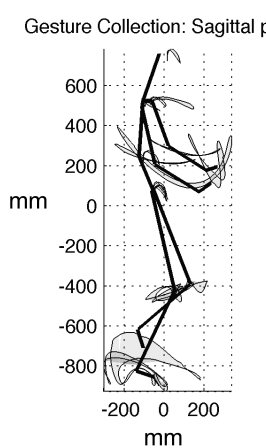

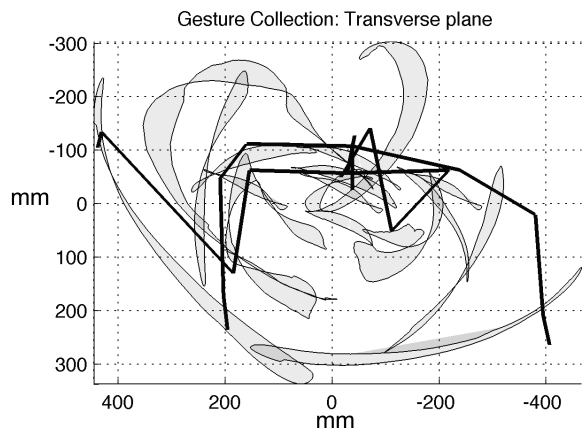

FIGURE 9. Basic Charleston gestures for different body joints (from a two-beat viewpoint). 


\section{Directional axis and basic gesture: professional samba dancer - right hand}
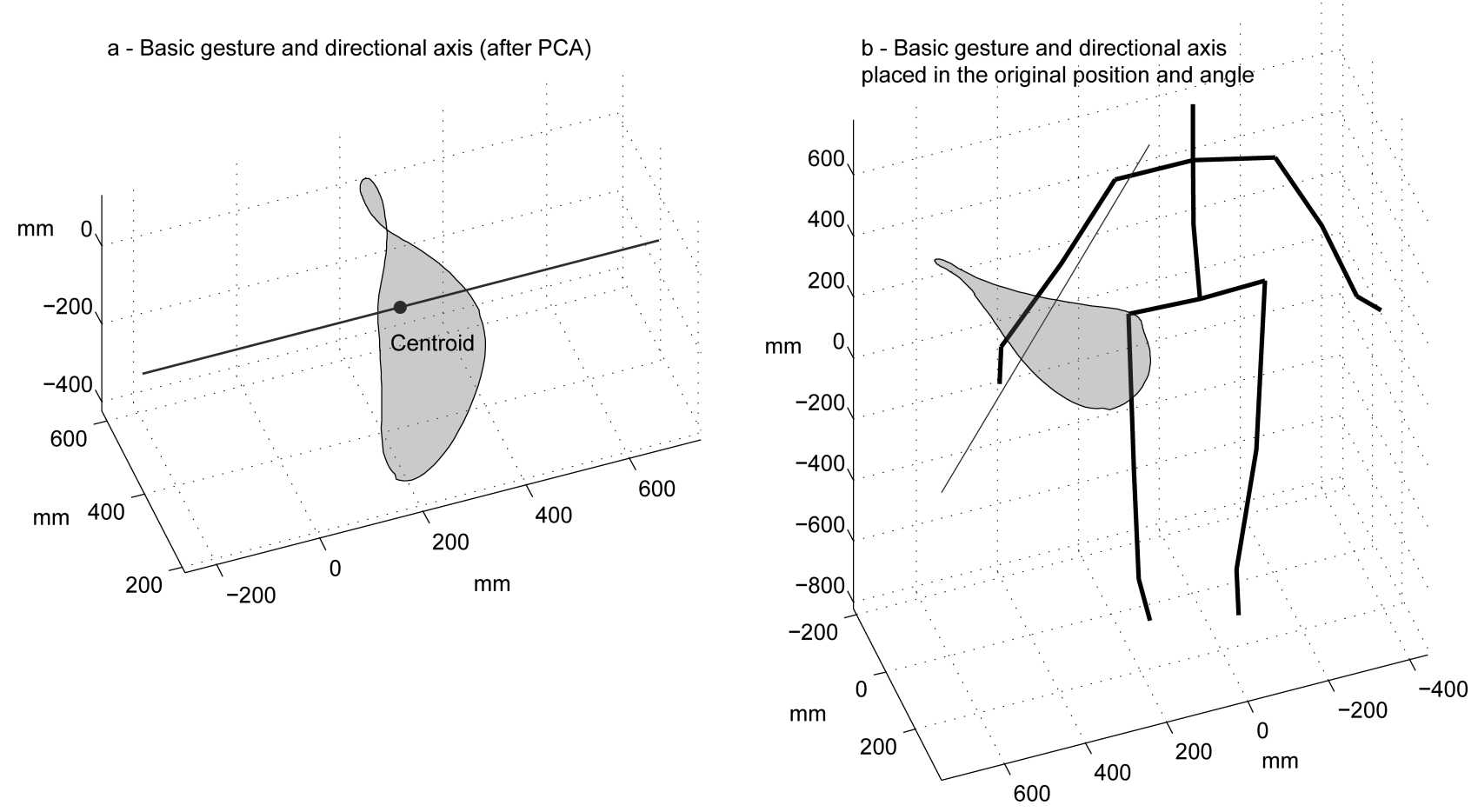

FIGURE 10. Representation of the gesture axis. (a) Basic gesture and perpendicular line through the centroid of the basic gesture, which represents the gesture axis. (b) The basic gesture and its gesture axis at its original position in relation to the representation of dancer's body.

superimposed in an arc-like shape. Feet gestures, which are characteristically ample in Charleston dances, demonstrate similar arc-like geometries. This similarity is reinforced by the gestures of the torso, which seem to be shaped by arched geometries as well. Again, like in samba, shapes of the torso and head are similar and symmetrical, even though the center-extremity trend found in the size of the basic gestures in samba is unclear in Charleston. In order to simplify the display, Figure 9 shows the basic gestures from the two-beat viewpoint. As mentioned, there are reasons to consider Charleston patterns from a four-beat viewpoint. This is of particular relevance for the foot patterns.

\section{The Gesture Axis}

The curved plane in which the gesture is represented can be linked in a direct way to the body-centered frame of reference. For that reason, it is straightforward to introduce the concept of a gesture axis; that is, a virtual axis that connects the body-centered perspective to the gesture. The gesture axis represents the body-centered perspective from which the control of the movement is likely to be most effective in terms of shape and variability. This representation of the gesture axis is obtained by plotting a line perpendicular to the first two principal components (or parallel to the third component), passing through the geometrical centroid of the basic gesture. Figure 10a shows the basic gesture and its axis as an isolated unit. Figure 10b shows this unit in conjunction with the skeleton of the dancer.

Figure 11 demonstrates the application of the concept of gesture axis to 16 joints on the body of the samba dancer (teacher). Interestingly, the slightly negative angle of the axis of most of the gestures, as seen from a twodimensional rotational viewpoint in the coronal place, seems to indicate an inclination bias of the whole body. In addition, the symmetry observed in the shapes of the basic gesture is repeated in the gesture axes. Figure 12 shows the same result for the Charleston dance, which seems to offer a contrast to the systematic symmetry found in both gestural shape and orientation in samba.

In Charleston dance, displayed in Figure 12, symmetries are not as clear as in samba, although some trends may be found. Given the fact that Charleston dances encompass larger whole body movements, including 
Basic gesture and Gesture axes: professional samba dancer
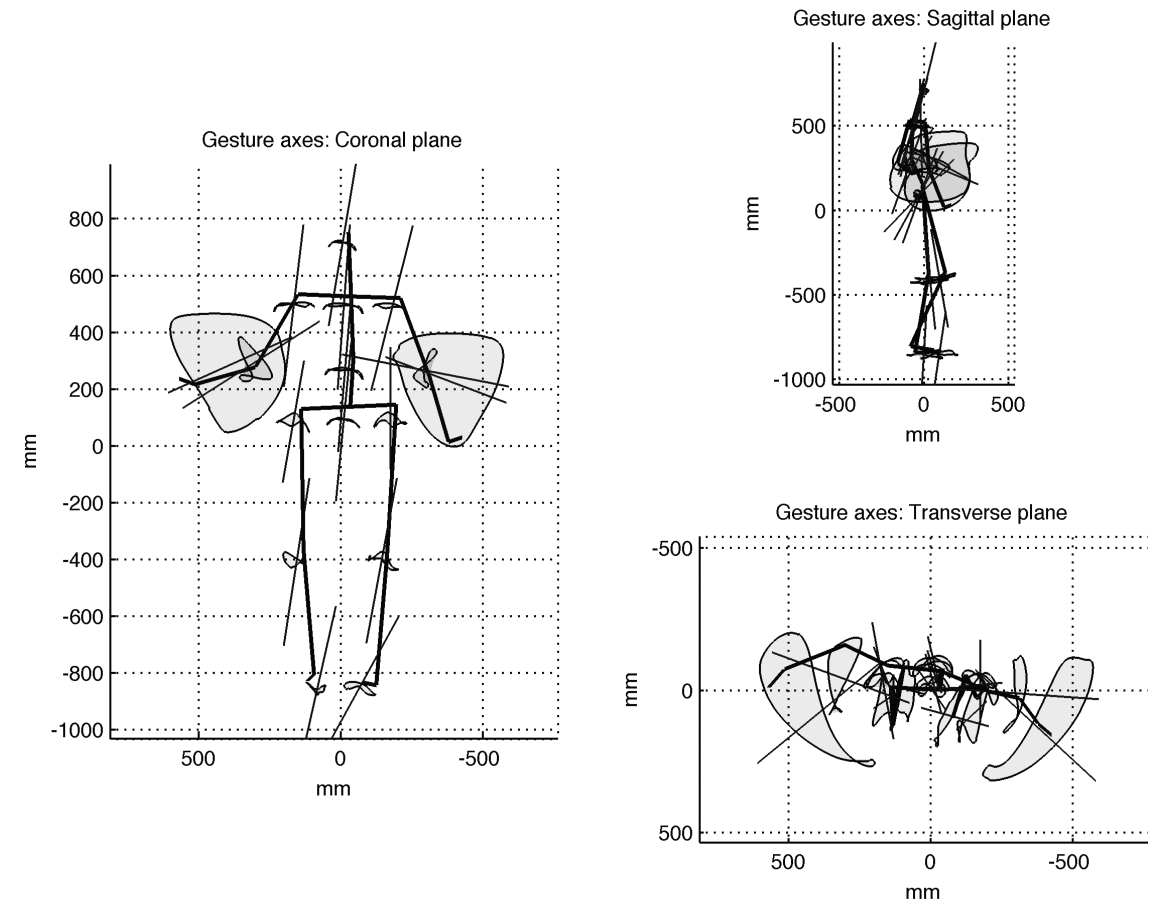

FIGURE 11. Representation of the dancer's body (professional samba dancer) displaying basic gestures and gesture axes for 16 joints.

Basic gesture and Gesture axes: professional Charleston dancer
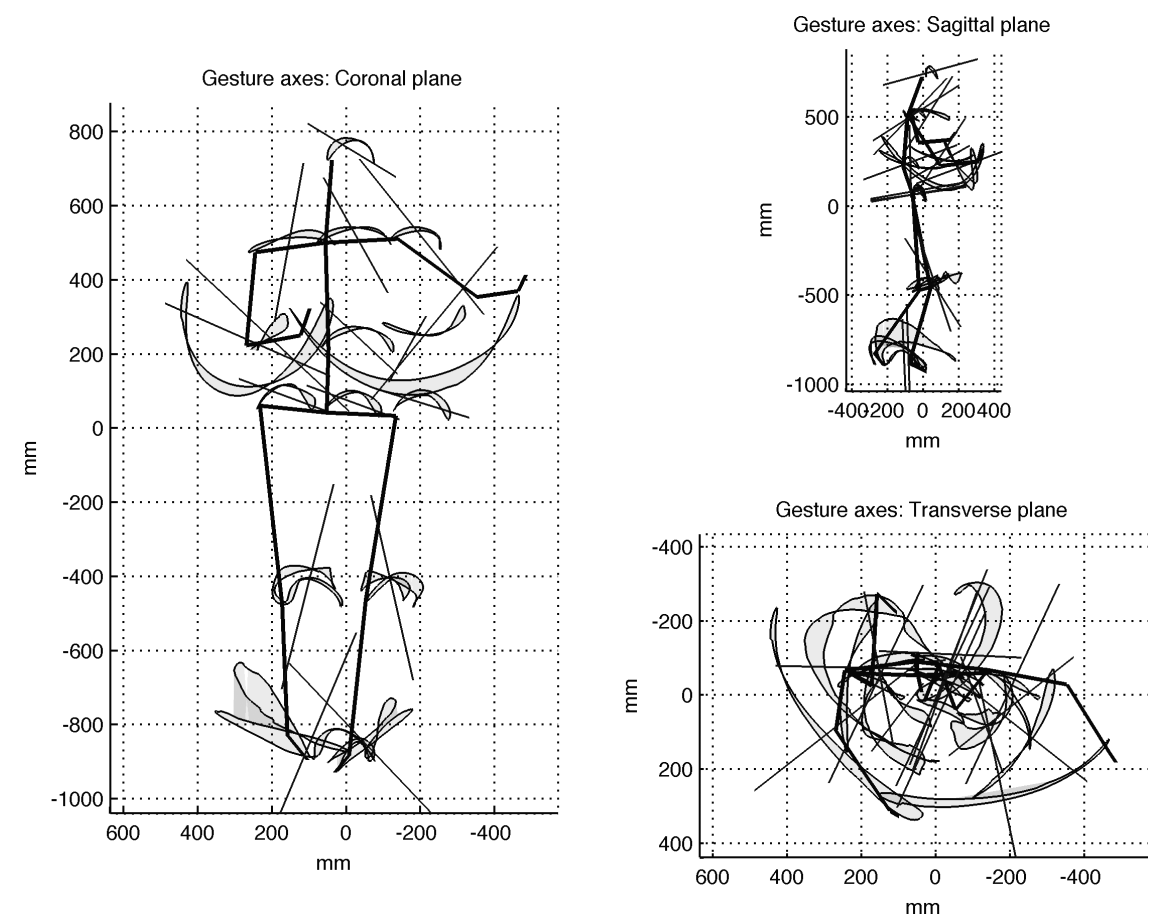

FIGURE 12. Representation of the dancer's body (professional Charleston dancer) displaying basic gestures and gesture axes for 16 joints. 


\section{Basic gesture and Gesture axes: student of samba}
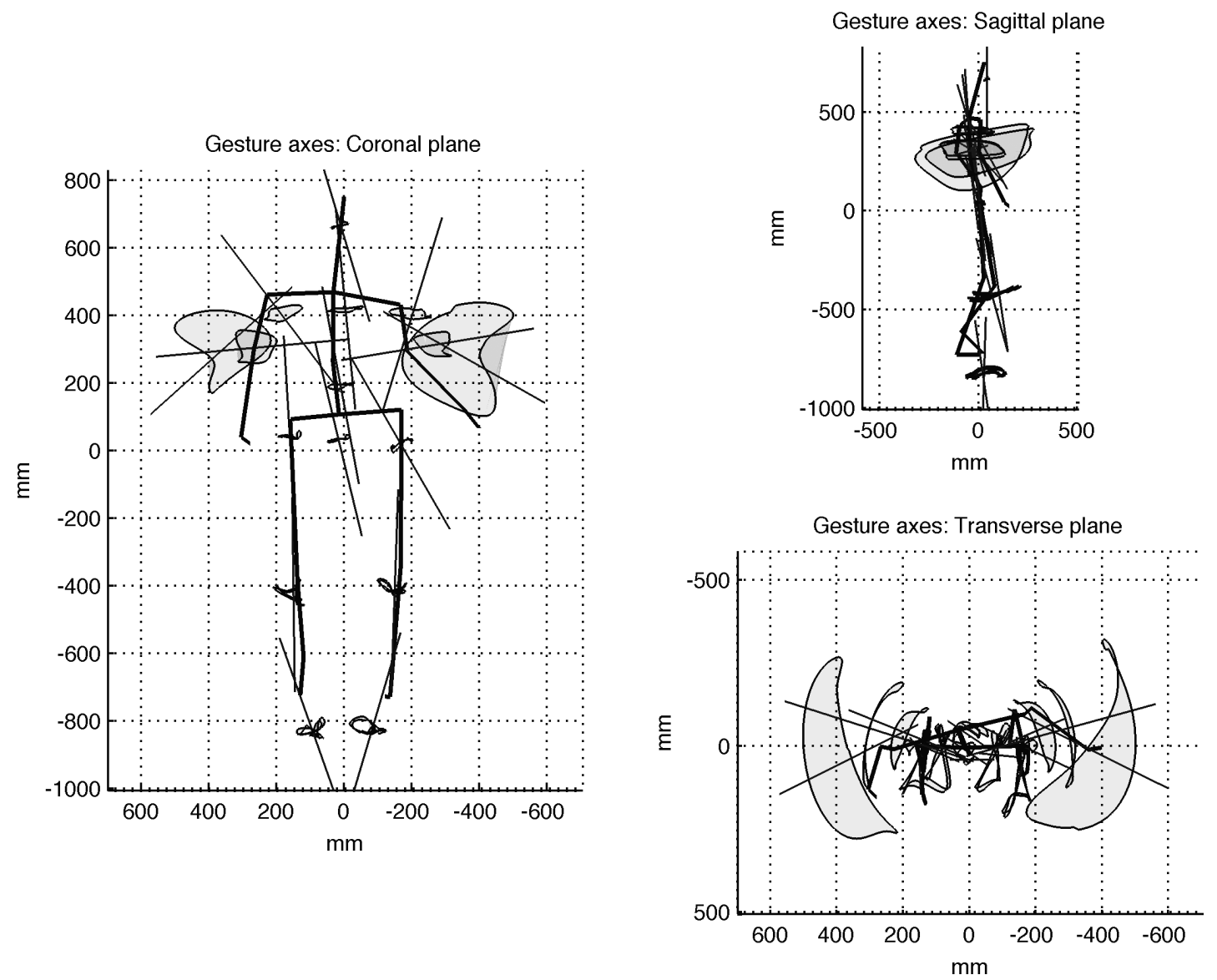

FIGURE 13. Stick figure and basic gesture of a samba dancer (student) with basic gestures and gesture axes for 16 joints.

jumps, the normalization of the trajectories may have imposed artifacts to the geometry of the gesture. Another explanation is that the dances or dancers' morphologies are simply different, or that the technical orientation of dance techniques is distinct. Further research is needed to better understand this issue.

The orientation of feet gestures is approximately perpendicular to the ground. The angle of hips and knees follow the bending tendencies of the leg, while head and shoulders are aligned with the torso. It is informative to compare the basic gesture map of the expert samba dancer in Figure 11 with the basic gesture map of the novice samba dancer of Figure 13.

Figure 13 shows the gesture axes of a student of samba dance. These axes seem to display less internal symmetry (symmetry between axes) than the gesture axes of the dancer teacher depicted in Figure 11. The differences may indicate that symmetry and parallelism between the directional angles of the gestures can play an important part in mastering stylistic forms in samba dances.
Linking Basic Gestures to Musical Features

The basic gesture, which captures the repetitive movement trajectory over time, can be superimposed with any musical cue derived from the synchronized audio signal. This is useful for studying the intricate relationship between dance movements and musical features. Two straightforward links are (i) time points, and (ii) loudness.

LINKING BASIC GESTURES TO TIME POINTS

The subdivision of the time domain vectors in discrete points representing metrical steps (e.g., 0.5 or 0.25 beats) will lead to a linear subdivision of the threedimensional space in the same metrical steps, which appear now as trajectory steps from one spatiotemporal reference point to another. The computation of this feature is rather simple since the sample position of any point in time will provide the position of the segmentation point in space. However, the procedure allows observing how meter unfolds as dance forms by indicating the 


\section{Basic gesture and metrical points: samba and Charleston}

\section{Charleston}
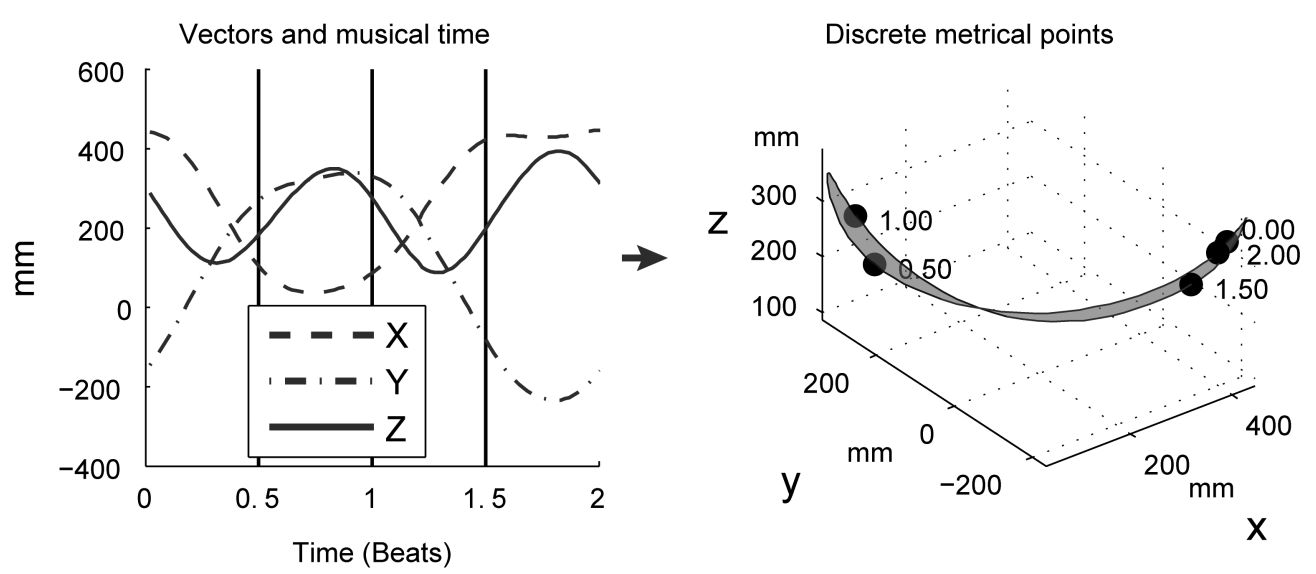

samba
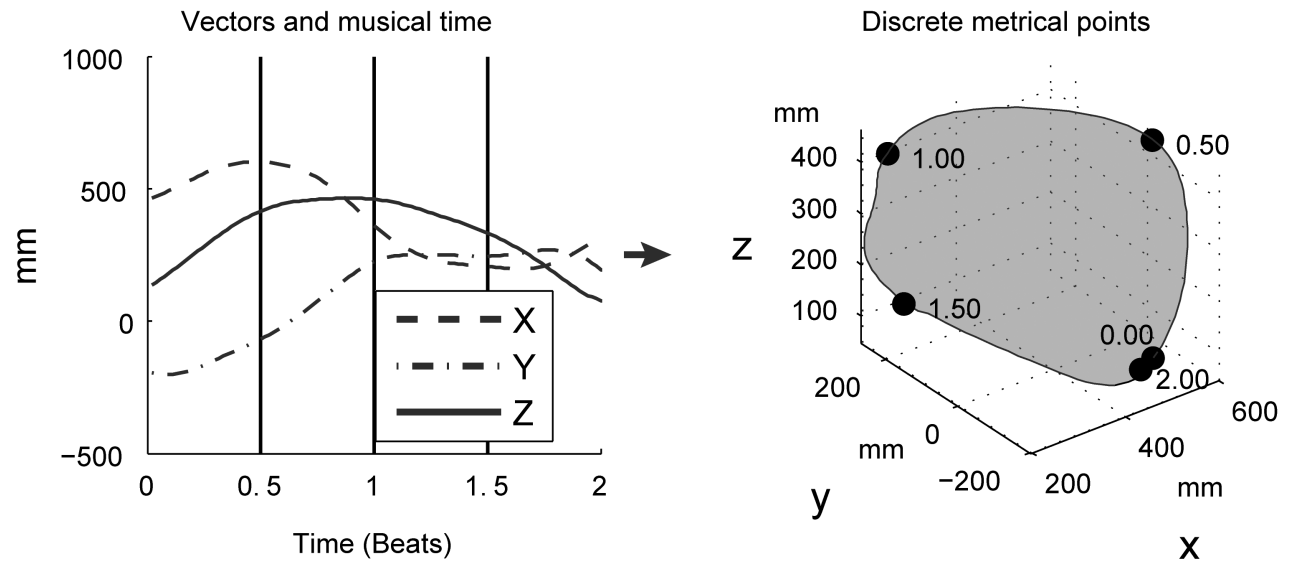

FIGURE 14. Linking basic gestures to musical time points. (a) The top panel displays the analysis of the right hand of the Charleston dancer. The left part shows the musical time points in the horizontal axis, and the position of in each dimensional component $X, Y$, and $Z$ in the vertical axis. The right part shows the reconstructed three-dimensional basic gesture with time points associated. (b) The bottom panel displays the same analysis, but for the right hand of the samba dance.

spatial locus, direction, and organization of musical meter along redundant trajectories. Figure 14 shows two examples of segmented gestures from samba and Charleston dances, both in the metrical level two-beats. The examples show the three-dimensional vectors that compose the bases of periodic gesture trajectories obtained from the periodicity analysis, segmented in steps of 0.5 beats and the concatenation of the three dimensions in a three-dimensional view.

This kind of visualization can unravel several interesting elements of the deployment of the dance gesture in time. The hand movement of the samba dancer, for example, signalizes each 0.5 beat step with a round and soft turn, making the gesture almost square-like form. It is possible to observe that the horizontal separation between signalization points is larger than the vertical separation of these points, which seem to indicate that metrical structures may be grouped in two horizontal metrical segments, $0.0-0.5$ and $1.0-0.5$. The hand movement of the Charleston dancer describes an arc gesture, but the beat or half beat points are not directly signalized by sharp turns in the movement. In this case, metrical points may be more clearly entrained by other body parts. This shows that metrical steps are very important elements of dance behavior, especially in popular forms of dance.

LINKING BASIC GESTURES TO LOUDNESS

Basic gestures can be linked with different features, such as acoustic loudness and instantaneous velocity. These features can be relevant to the understanding of dance/ music relationships. 


\section{Basic gesture and loudness: professional samba dancer (right hand)}
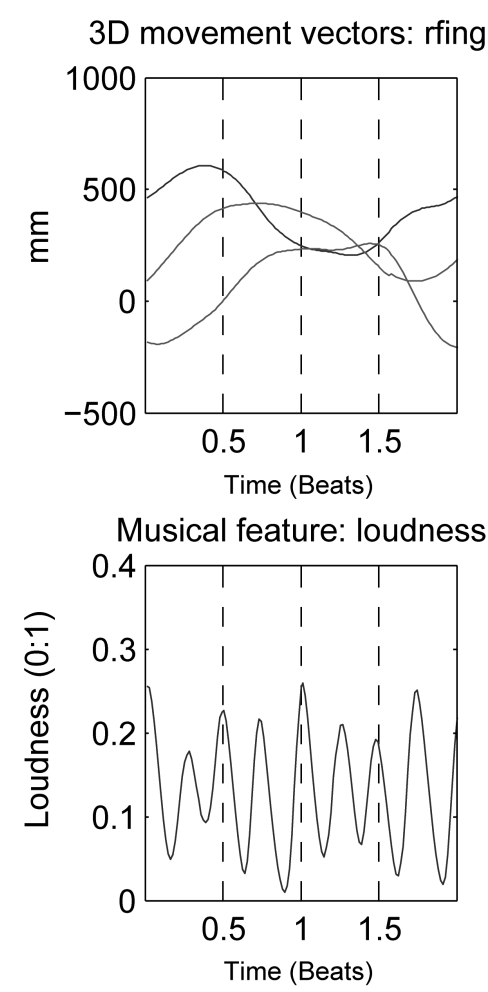

Distributed musical feature along gesture Musical Loudness

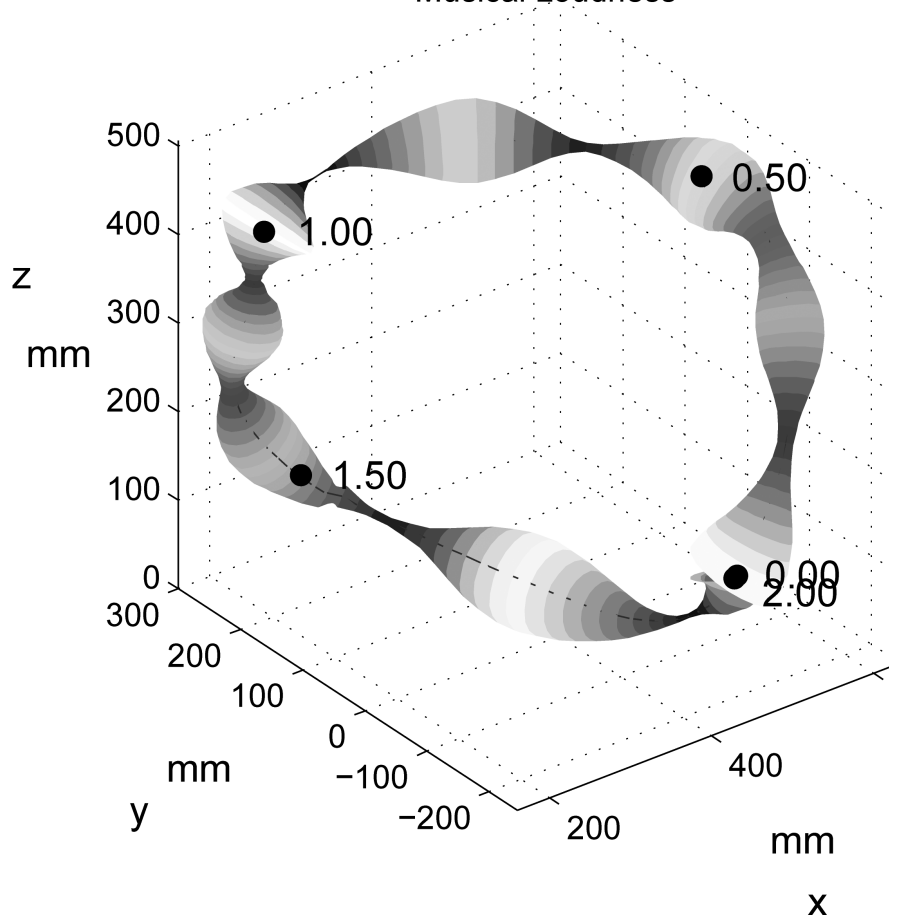

FIGURE 15. Tube plot of the basic gesture (samba dancer's right hand) with respective analysis of loudness patterns.

We developed a visualization based on the tubeplot scripts for Matlab from Sandberg (2009) that provides an example of how gesture trajectories offer a basis for crossmodal visualizations. In the example shown in Figure 15, the radius and the color shading of the transparent tube around the basic gesture are controlled by continuous variables, while the shape is defined by the basic gesture of the right hand (samba dancer). For visualization purposes in this example, the radius and color shading (in gray-scale) are controlled by the same variable. The idea behind this visualization is to add visual cues (radius and color shading) for the observation of interactions between spatial description of gestures and other external variables. Obviously, the representation of external variables is explorative and symbolic, as these variables are not related to the physical space around the gesture. Note that the time represented here was previously transformed into a "periodic space" because the heuristics of the periodicity analysis projected the movement signals onto a periodic subspace. Therefore, continuous variables can only match this periodic time if they are an excerpt of the signal that fits the time of the periodicity (e.g., the first two beats of the signal), or if they are also periodicities, which were projected onto an identical periodic subspace (a subspace with the same period). In Figure 15, we projected the periodicity basis for loudness of the audio of the stimuli played during the experiment. We projected the loudness curves onto a subspace of two-beat period. The loudness curves were calculated using the IPEMtoolbox (Leman, Lesaffre, \& Tanghe, 2001).

The periodicity of loudness patterns (continuous variable) controls the radius and color shading (black:white $=0: 1)$ of the tube around the gesture path. The scale between the radius and the feature description must be defined manually because the connection between the physical quantities (e.g., radius in millimeters, loudness in ratio) in the representation is symbolic. The uses of logarithmic scales and other optimizations may be taken into consideration to improve the readability of the representation.

The representation produces the overall geometry of the gesture and introduces important information about the stimuli. For example, strong loudness onset periodicities seem to be placed at the time points where the gesture reaches the horizontal extremities of the spatial geometry. 


\section{Basic gesture and speed: professional samba dancer (right hand)}
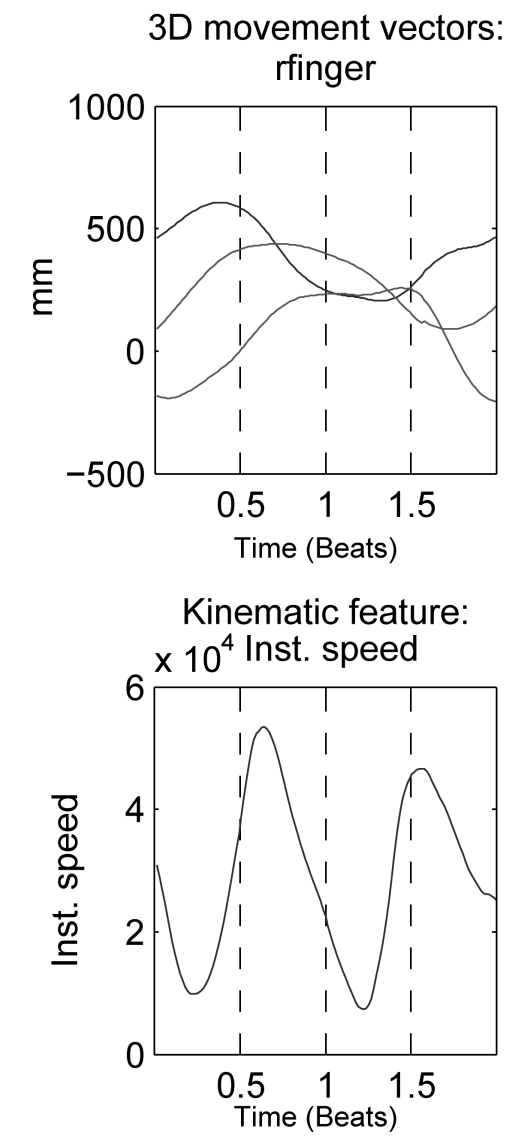

(M.level: 2 beats) Instantaneous speed

FIGURE 16. Dance gesture and representation of instantaneous speed.

These loudness onsets induce syncopation forces, while movement patterns seem to signalize metrical elements.

\section{Linking Basic Gestures to Kinematic Features}

In a similar way as with timing and loudness, it is possible to map kinematic features onto the basic gesture. Figure 16 displays the instantaneous speed derived from the same periodic trajectories plotted against the gesture form. In this figure, we observe, on the one hand, that the speed of the gesture decreases as in the vicinity of the beat markers. Changes in speed require the use of more muscular energy to stop and reinitiate displacement. On the other hand, the lower speed observed in the beat points indicates a tendency to "rest" at the beat points. Note that when the first beat starts, the instantaneous speed grows more gradually, probably because of the gravitational force, which reduces the speed retake in the ascendant movement.

\section{General Discussion}

The idea that basic gestures are spatiotemporal frames of reference for action-perception couplings can be related to studies that consider body-centered representations (though not necessarily from the viewpoint of dance/music couplings). Studies in phenomenology (e.g., Merleau-Ponty, 1945) and ecological perception (e.g., Gibson, 1979) were the first to draw attention to the fact that the cognition of space may be strongly dependent on the subject's action-oriented perception of the environment, suggesting that the representation of space is highly subjective and multifaceted. In this context, Previc (1998) distinguishes four major spaces, namely: (a) the peripersonal space, which is accessed by operations in the near-body space (similar to Laban's kinesphere); (b) the focal extrapersonal space, which is accessed through visual search and object recognition; (c) the action-extrapersonal space, which is accessed by 
orientation in a space containing referential landmarks or points; and (d) the ambient-extrapersonal space, which is characterized by orientation in an earth-fixed coordinate system. A related distinction can be made between egocentric representations, in which locations are represented with regards to the body-centered (eye-, head-, and arm-centered) reference frames, and allocentric representations, where reference frames for spatial localization are environment-centered, including those centered in an object of interest (Ghafouri \& Lestienne, 2006). Additional characterizations are the egocentric space, or body space that is covered by our skin, or the back space or no-motor space, of which we are aware but cannot reach or see (Saj \& Vuilleumier, 2007).

The study of peripersonal space is highly relevant to the problem of how dance and music are dealt with in terms of representations (cf. Ghafouri \& Lestienne, 2006). The concept of peripersonal space seems to imply a representation that depends on a body-centered orientation of the action (Ghafouri \& Lestienne, 2006; Gourtzelidis, Smyrnis, Evdokimidis, \& Balogh, 2001). However, this representation would consist of multiple frames of bodycentered reference (Caggiano, Fogassi, Rizzolatti, Thier, \& Casile, 2009), and arm length may constitute an intrinsic metric for the representation of near space (Longo \& Lourenco, 2007). In that perspective, basic gestures for dance can be considered to result from multimodal integration of visual, vestibular, somatosensory, and auditory signals (Hidot, Lafaye, \& Saint-Jean, 2006; Lepelley, Thullier, Koral, \& Lestienne, 2006; Longstaff, 2000), and the spatiotemporal reference frame can be considered from the viewpoint of spatial (body-centered) occupations (e.g., close or at a distance from the body-center). Although the studies on peripersonal space reveal different conceptions about the cognition of space, they all somehow rely on the idea that spatial cognition involves a subjective frame of reference that implies a bodycentered viewpoint and an action-based orientation. The concept of basic gesture may be helpful in understanding the action-perception couplings of dance and music. However, further research will be needed to better understand and model the peripersonal representation in relation to the biomechanics of the human body, including rotation of the shoulders with respect to the body center. The present model of normalization may be limited to the kind of repetitive dance patterns that involve little rotation, such as the ones collected in the present study. In addition, further study is needed about the role of auditory cues in movement boosts. Styns et al. (2007) found that in walking on music, subjects walk faster on music than on metronome ticks at the same tempo. This suggests that several acoustical parameters, such as pitch, accent, loudness - which occur at specific points in time-may induce movement.

It is tempting to assume that spatiotemporal reference frames, which are based on auditory cues and eigenfrequencies of human body movement, may play a role as regulating mechanisms for the synchronization (tempo correspondence) and entrainment (phase correspondence) (Clayton, Sager, \& Will, 2004) of action patterns in relation to music. It is furthermore important to note that the embodiment of music does not imply that the dancer would only mimic musical gestures through body gestures, as several authors have drawn attention to the fact that movement and gestures in samba contexts can be in counterpoint to the samba musical gestures (Browning, 1995; Sandroni, 2001; Sodré, 1979).

Although the present paper offers a method that extracts basic gestures from actually executed repetitive dance patterns, we believe that basic gestures can be conceived as frames of reference in the mental and motor domain, as was suggested in the introduction part of this paper (Brown, Martinez, \& Parsons, 2006; Farnell, 1994; Gallagher, 2005; Leman, 2007; Metzinger, 2000; Sandroni, 2001; Tversky, 2003). Several authors have used the term body schema to denote a 'muscle memory' that guides the position of body parts with respect to one another over time, resulting in an internal dynamic model of the body (Buxbaum, Giovannetti, \& Libon, 2000). In parallel, the term body image has been used to denote the conceptual knowledge about this guidance (Gallagher, 2005; Metzinger, 2000). Apart from the deployed basic gesture as a physically repetitive dance pattern, we believe that it is straightforward to conceive basic gestures as body schemata and/or body images that guide dance and music couplings in the motor and mental domain. This can also be linked to information theoretical and physiological studies of action perception couplings, where reference frames are assumed to play a role in guided action. For example, Lee (1998) has proposed a theory that deals with the description of the gap between a current movement state of a body part and a goal state. In this approach, basic gestures may function as spatiotemporal frames of reference for the guided motion and coupling of body parts (which can be mental-based or more motor-based).

In the threshold control theory (Feldman, 2009; Feldman \& Levin, 2009), a basic gesture could accomplish a reference frame that is defined by the sensory inputs to neurons, of which the reference threshold for minimal effort of movement can be modified in a feedforward way, in order to intentionally control the action. The theory takes into account the role of sensory modes 
in combination with representational modes (Paillard, 1991), which reflects the view that basic gestures provide frames for matching action and perception. In that perspective, a basic gesture is consistent with the idea of a memory for condition-specific thresholds shifts that handle reference frames that match action and perception. In that context, the reference to minimal effort takes on a significant importance in repetitive dances. We believe that dancers may obtain maximal rendition of gestures with minimal effort when the movements are close to the eigenfrequency of the body part. Further research is needed to conceive ways in which shifts from one gesture to the other gesture can be represented, as well as how the gestures can be nested into larger memory frames that make up entire choreographies.

\section{Conclusion}

The present study contributes to the idea that dancers perform music-driven repetitive dance patterns using spatiotemporal frames of reference, called basic gestures, that couple perceived musical cues with the movement of body parts. Technically speaking, these frames of reference can be conceived as spatial geometries onto which musical cues (and by extension also body cues) can be projected. Conceptually speaking, the spatiotemporal reference frames control minimum effort points in action-perception couplings. They reside as memory patterns in the mental and/or motor domains, ready to be dynamically transformed in dance movements.

The supporting evidence in favor of the concept of basic gesture is based on an approach where basic gestures are extracted from repetitive dance patterns. This approach is based on several assumptions that define and confine the concept of basic gesture; such as the idea of a body-centered frame of reference, embodiment of musical cues in dance gestures, minimal effort in relation to spherical spaces and resonance frequencies, the direction of the so-called gesture axis, and so on. It cannot be denied that, given these assumptions, and given the limited amount of subjects that have been observed so far, the proposed concept of basic gesture is still in an early stage, and that more work is needed that should contribute to a refinement of the concept.

\section{Author Note}

The authors wish to thank Chris Müller and Leon van Noorden for useful comments on a first draft of the paper.

Correspondence concerning this article should be addressed to Marc Leman, IPEM, Blandijnberg 2, 9000 Ghent, Belgium. E-MAIL: marc.leman@ugent.be

\section{References}

BECKING, G. (1928). Der musikalische rhythmus als erkenntnisquelle [Musical rhythm as a source of insight]. Augsburg: B. Filser.

Bevilacqua, F., Ridenour, J., \& Cuccia, D. (2002). 3d motion capture data: Motion analysis and mapping to music. In Q. Stout \& M. Wolfe (Eds.), The Sixth Distributed Memory Computing Conference (pp. 562-569). Santa Barbara, CA: IEEE Computer Society Press.

Brown, S., Martinez, M. J., \& Parsons, L. M. (2006). The neural basis of human dance. Cerebral Cortex, 16, 1157-1167.

Browning, B. (1995). Samba: Resistance in motion. Bloomington, IN: Indiana University Press.

Buxbaum, L., Giovannetti, T., \& Libon, D. (2000). The role of the dynamic body schema in praxis: Evidence from primary progressive apraxia. Brain and Cognition, 44, 166-191.

Caggiano, V., Fogassi, L., Rizzolatti, G., Thier, P., \& CAsile, A. (2009). Mirror neurons differentially encode the peripersonal and extrapersonal space of monkeys. Science, $324,403$.
CAMURRI, A. (2002). Motion analysis library reference (Version 2.0). Genova: Laboratorio di Informatica Musicale-DIST.

Camurri, A., Lagerlof, I., \& Volpe, G. (2003). Emotions and cue extraction from dance movements. International Journal of Human Computer Studies, 59, 213-225.

Camurri, A., Mazzarino, B., \& Volpe, G. (2004). Analysis of expressive gesture: The eyesweb expressive gesture processing library. In A. Camurri \& G. Volpe (Eds.), Gesture-based communication in human-computer interaction (Vol. 2915, pp. 460-467). Heidelberg, Germany: Springer Verlag.

Clayton, M., Sager, R., \& Will, U. (2004). In time with the music: The concept of entrainment and its significance for ethnomusicology. ESEM Counterpoint, 1, 1-82.

Counsell, C. (2006). The kinesics of infinity: Laban, geometryand the metaphysics of dancing space. Dance Research, 24, 105-116.

De Bruyn, L., Leman, M., \& Moelants, D. (2009). Quantifying children's embodiment of musical rhythm in individual and group settings. In Y. K. Miyazaki (Ed.), Tenth International Conference on Music Perception and Cognition (pp. 662-667). Sapporo, Japan: International Society for Music Information Retrieval. 
De Bruyn, L., Leman, M., Moelants, D., \& Demey, M. (2009). Does social interaction activate music listeners? In S. Ystad, R. Kronland-Martinet, \& K. Jensen (Eds.), Computer music, modeling and retrieval. Genesis of meaning of sound and music. Lecture notes in computer science. (Vol. 5493, pp. 93-106). Berlin, Germany: Springer-Verlag.

Eerola, T., Luck, G., \& Toiviainen, P. (2006). An investigation of pre-schoolers' corporeal synchronization with music. In M. Baroni, A. R. Addessi, R. Caterina, \& M. Costa (Eds.), Ninth International Conference on Music Perception and Cognition (pp. 472-476). Bologna, Italy: ICMPC and ESCOM.

FARNell, B. (1994). Ethno-graphics and the moving body. Man, 4, 929-974.

Feldman, A. (2009). New insights into action-perception coupling. Experimental Brain Research, 194, 39-58.

Feldman, A. G., \& Levin, M. F. (2009). The equilibriumpoint hypothesis: Past, present and future. In D. Sternad (Ed.), Progress in motor control: A multidisciplinary perspective (Vol. 629, pp. 699-726). New York: Springer.

Gallagher, S. (2005). Body schema and intentionality. In J. Bermudez, A. Marcel, \& N. Eilan (Eds.), The body and the self (pp. 225-244). Cambridge, MA: MIT/Bradford Press.

Ghafouri, M., \& Lestienne, F. (2006). Contribution of reference frames for movement planning in peripersonal space representation. Experimental Brain Research, 169, 24-36.

GiBson, J. (1979). The ecological approach to visual perception. Boston, MA: Houghton Mifflin.

Glowinski, D., Camurri, A., Chiorri, C., Mazzarino, B., \& VOLPE, G. (2009). Validation of an algorithm for segmentation of full-body movement sequences by perception: A pilot experiment. In M. Sales Dias, S. Gibet, M. M. Wanderley, \& R. Bastos (Eds.), Gesture-based human-computer interaction and simulation (Vol. 5085, pp. 239-244). Berlin, Germany: Springer-Verlag.

Godøy, R. I., \& Leman, M. (2010). Musical gestures: Sound, movement, and meaning. New York: Routledge.

Gourtzelidis, P., Smyrnis, N., Evdokimidis, I., \& Balogh, A. (2001). Systematic errors of planar arm movements provide evidence for space categorization effects and interaction of multiple frames of reference. Experimental Brain Research, 139, 59-69.

GUEDES, C. (2006). Extracting musically-relevant rhythmic information from dance movement by applying pitchtracking techniques to a video signal. In A. Baratè, G. Haus, \& L. A. Ludovico (Eds.), Proceedings of the Sound and Music Computing Conference SMC06 (pp. 25-33). Marseille: GMEM.

Hidot, S., Lafaye, J., \& Saint-Jean, C. (2006). Discriminant factor analysis for movement recognition: Application to dance. Machine Graphics and Vision, 15, 391.

Laban, R., \& LaWrence, F. (1947). Effort. London: MacDonald and Evans.
Laban, R., \& Ullmann, L. (1966). Choreutics. London: MacDonald and Evans.

Laban, R., \& Ullmann, L. (1980). The mastery of movement. London: MacDonald \& Evans.

LeE, D. (1998). Guiding movement by coupling taus. Ecological Psychology, 10, 221-250.

LEMAN, M. (2007). Embodied music cognition and mediation technology. Cambridge, MA: MIT Press.

LEMAN, M., \& GODøY, R. I. (2010). Why study musical gestures? In R. I. Godøy \& M. Leman (Eds.), Musical gestures: Sound, movement, and meaning (pp. 3-11). New York: Routledge.

Leman, M., Lesaffre, M., \& TANGHe, K. (2001). A toolbox for perception-based music analyses [Manual].Ghent, Belgium: IPEM-Department of Musicology, Ghent University.

Lepelley, M., Thullier, F., Koral, J., \& Lestienne, F. (2006). Muscle coordination in complex movements during jete in skilled ballet dancers. Experimental Brain Research, 175, 321-331.

London, J. (2006). Musical rhythm: Motion, pace and gesture. In A. Gritten \& E. King (Eds.), Music and gesture (pp. 126141). Burlington, VT: Ashgate Publishing Company.

LONGO, M., \& LOURENCO, S. (2007). Space perception and body morphology: Extent of near space scales with arm length. Experimental Brain Research, 177, 285-290.

LONGSTAFF, J. (2000). Re-evaluating rudolf laban's choreutics. Perceptual and Motor Skills, 91, 191-210.

MacDougall, H. G., \& Moore, S. T. (2005). Marching to the beat of the same drummer: The spontaneous tempo of human locomotion. Journal of Applied Physiology, 99, 1164-1173.

Merleau-Ponty, M. (1945). Phénoménologie de la perception [Phenomenology of perception]. Paris: Edition Gallimard.

MetZinger, T. (2000). The subjectivity of subjective experience: A representationalist analysis of the first-person perspective. In T. Metzinger (Ed.), Neural correlates of consciousness: Emprical and conceptual questions (pp. 285-306). Cambridge, MA: MIT Press.

Naveda, L., \& Leman, M. (2008a). Representation of samba dance gestures, using a multi-modal analysis approach. In E. Ruffaldi \& M. Fontana (Eds.), ENACTIVE08 - 5th International Conference on Enactive Interfaces (pp. 68-74). Pisa, Italy: Edizione ETS.

NaVEDA, L., \& Leman, M. (2008b). Sonification of samba dance using periodic pattern analysis. In À. Barbosa (Ed.), ARTECH 2008. 4th International Conference on Digital Arts (pp. 16-26). Porto, Portugal: Universidade Catolica Portuguesa.

NaVeda, L., \& Leman, M. (2009). A cross-modal heuristic for periodic pattern analysis of samba music and dance. Journal of New Music Research, 38, 255-283.

Nettheim, N., \& BeCKing, G. (1996). How musical rhythm reveals human attitudes: Gustav Becking's theory. International Review of the Aesthetics and Sociology of Music, 27, 101-122. 
Paillard, J. (1991). Motor and representational framing of space. Brain and Space, 163-182.

Phillips-Silver, J., \& Trainor, L. J. (2007). Hearing what the body feels: Auditory encoding of rhythmic movement. Cognition, 105, 533-546.

Previc, F. (1998). The neuropsychology of 3-d space. Psychological Bulletin, 124, 123-164.

SAJ, A., \& Vuilleumier, P. (2007). Neglect: Remembering the space left behind. Current Biology, 17, 1060-1062.

Sandberg, A. (2009). Tubeplot [Matlab script]. Retrieved from http://www.nada.kth.se/ asa/Ray/Tubeplot/tubeplot.html

SAndroni, C. (2001). Feitiço decente: Transformações do samba no Rio de Janeiro, 1917-1933 [Decent sorcery: Transformations of the samba in Rio de Janeiro]. Rio de Janeiro: Jorge Zahar Editor/Editora UFRJ.

Sethares, W. A., \& Staley, T. W. (1999). Periodicity transforms. IEEE Transactions on Speech and Audio Processing, 47, 2953-2964.

Shiratori, T., Nakazawa, A., \& Ikeuchi, K. (2003). Rhythmic motion analysis using motion capture and musical information. Proceedings of the IEEE Conference on Multisensor Fusion and Integration for Intelligent Systems (Vol. 5, pp. 89-94). Tokyo, Japan: University of Tokyo.

SOdré, M. (1979). Samba, o dono do corpo [Samba: The owner of the body]. Rio de Janeiro: Codecri.
Styns, F., van Noorden, L., Moelants, D., \& Leman, M. (2007). Walking on music. Human Movement Science, 26, 769-785.

Toiviainen, P., \& Burger, B. (2010). MoCap Toolbox Manual. University of Jyväskylä: Jyväskylä, Finland. Available at http://www.jyu.fi/music/coe/materials/mocaptoolbox/ MCTmanual

TVersky, B. (2003). Structures of mental spaces: How people think about space. Environment and Behavior, 35, 66-79.

VAn Noorden, L. (2010). The functional role and biokinetics of basic and expressive gestures in activation and sonification. In R. I. Godøy \& M. Leman (Eds.), Musical gestures: Sound, movement, and meaning (pp. 154-179). New York: Routledge.

Van Noorden, L., \& De Bruyn, L. (2009). The development of synchronisation skills of children 3 to 11 years old. In P. Toivanen (Ed.), ESCOM-7th Triennial Conference of the European Society for the Cognitive Sciences of Music. Jyväskylä, Finland: University of Jyväskylä.

Van Noorden, L., \& Moelants, D. (1999). Resonance in the perception of musical pulse. Journal of New Music Research, $28,43-66$.

YAмамото, T., \& Fujinami, T. (2008). Hierarchical organization of the coordinative structure of the skill of clay kneading. Human Movement Science, 27, 812-822. 
